\title{
Algebraic Quantization of Systems with a Gauge Degeneracy
}

\author{
Hendrik B. G. S. Grundling and C. A. Hurst \\ Department of Mathematical Physics, University of Adelaide, South Australia 5001
}

\begin{abstract}
Systems with a gauge degeneracy are characterized either by supplementary conditions, or by a set of generators of gauge transformations, or by a set of constraints deriving from Dirac's canonical constraint method. These constraints can be expressed either as conditions on the field algebra $\mathscr{F}$, or on the states on $\mathscr{F}$. In a $C^{*}$-algebra framework, we show that the state conditions give rise to a factor algebra of a subalgebra of the field algebra $\mathscr{F}$. This factor algebra, $\mathscr{R}$, is free of state conditions. In this formulation we show also that the algebraic conditions can be treated in the same way as the state conditions. The connection between states on $\mathscr{F}$ and states on $\mathscr{R}$ is investigated further within this framework, as is also the set of transformations which are compatible with the set of constraints. It is also shown that not every set of constraints can give rise to a nontrivial system. Finally as an example, the abstract theory is applied to the electromagnetic field, and this treatment can be generalized to all systems of bosons with linear constraints. The question of dynamics is not discussed.
\end{abstract}

\section{Introduction}

The meaning of the term "systems with a gauge degeneracy" that we refer to is that of systems with a mathematical degree of freedom that is not physically realized, i.e. a mathematical redundancy. The problem then is to find a canonical procedure that will rid the system of this redundancy, leaving only the physical theory.

The problem of gauge degeneracies occurs in classical systems as early as the variational formulation, where it appears as the problem of the non-uniqueness of solutions in Hamilton's principle, [1]:

$$
\delta S[q]=\delta \int_{s}^{t} L(q, \dot{q}, t) d t=0 .
$$

Here, $q(t)$ is the path of motion between times $s, t$. It is found that there are an infinite number of solutions if $S^{\prime \prime}[q]=0$, where $S^{\prime}[q]$ denotes the Fréchet derivative 
of $S$ with relation to path space [1]. This means $\left(\partial^{2} L / \partial \dot{q}^{2}\right)=0$, or in the case of more than one degree of freedom

$$
\operatorname{det}\left[\frac{\partial^{2} L}{\partial \dot{q}_{i} \partial \dot{q}_{j}}\right]=\operatorname{det}\left[\frac{\partial p_{i}}{\partial \dot{q}_{j}}\right]=0,
$$

and this is just the condition characterizing the class of "singular Lagrangians" to which Dirac's canonical constraint procedure is applicable [2-6].

In its naivest form, Dirac's constraint theory is as follows. The singularity condition (1.1) indicates immediately that the Lagrangian formalism $\left(t, q_{i}, \dot{q}_{i}\right)$, $i=1, \ldots, N$, is not equivalent to the Hamiltonian formalism $\left(t, q_{i}, p_{i}\right), i=1, \ldots, N$. Indeed, let the rank of the matrix $\left[\partial p_{i} / \partial \dot{q}_{j}\right]$ be $R, R<N$. Then in passing from $\left(t, q_{i}, p_{i}\right)$ to $\left(t, q_{i}, \dot{q}_{i}\right)$, the system of equations $p_{i}=p_{i}(q, \dot{q}, t), i=1, \ldots, N$ can only be solved for $R$ velocities, and there are $N-R$ independent relations $\psi_{s}(q, p)=0$, called primary constraints. The effect of these is to reduce the phase space $\left(q_{i}, p_{i}\right)$, $i=1, \ldots, N$ to an $N+R$ dimensional manifold $Y$. From the requirement that all dynamical trajectories should lie in this manifold, we get the consistency conditions: $\dot{\psi}_{s}(q, p) \uparrow Y=\left\{\psi_{s}, H\right\} \uparrow Y=0$, where $\{$,$\} is the Poisson bracket and H$ is the Hamiltonian. The requirement for these to hold leads to a new set of constraints, which in turn should also satisfy consistency conditions on the further reduced manifold. The process is continued until it terminates, and in general an additional set of constraints $\left\{\varphi_{s}(q, p)\right\}$, called secondary constraints, are obtained. The total set of constraints, denoted $\left\{\chi_{k}\right\}$, defines the "reduced phase space" $Z$. The set of phase space functionals $P$ can now be divided into two sets, first we have $\mathcal{O}_{c}=\left\{A \in P ;\left\{A, \chi_{k}\right\}\{Z=0 \forall k\}\right.$, called the observables or first class variables or weak commutant of $\left\{\chi_{k}\right\}$, and second the set of gauge variables $P \backslash \mathcal{O}_{c}$. Then the first class constraints are the elements of $\left[\left\{\chi_{k}\right\}\right] \cap \mathcal{O}_{c}=.\left\{\alpha_{s}\right\}$, where $[\cdot]$ denotes the linear space generated by its argument, and the second class constraints are $\left\{\xi_{r}\right\}=\left[\left\{\chi_{k}\right\}\right] \backslash\left\{\alpha_{s}\right\}$. By taking linear combinations we can obtain a maximum number of first class constraints. Originally $P$ formed a function group under the Lie product defined by the Poisson bracket. Since the phase space has been reduced to $Z$, a new Lie product will have to be defined on the set of functionals on $Z$ to ensure that this set will be a function group. This Lie product is the Dirac bracket, $[6,7]:\{A, B\}_{D}=\{A, B\}-\left\{A, \xi_{r}\right\} C_{r s}\left\{\xi_{s}, B\right\}$, where $C_{r s}=\left[\left\{\xi_{k}, \xi_{l}\right\}\right]_{r s}^{-1}$, and use is made of the summation convention. All second class constraints vanish identically with relation to the Dirac bracket. Furthermore, since the Dirac bracket satisfies the Jacobi identity, is bilinear and antisymmetric, it is a suitable ansatz for the quantum mechanical commutator. It is possible to transform to a natural coordinate system on $Z$, so that in this coordinate system the Dirac bracket will become a Poisson bracket, [7]. The dynamical equations become: $\dot{F}\left\lceil Z=\left\{F, H_{E}\right\}_{D}\left\lceil Z+(\partial F / \partial t)\left\lceil Z\right.\right.\right.$, where the extended Hamiltonian $H_{E}$ is defined by $H_{E}=H+\lambda_{\alpha} \chi_{\alpha}, \lambda_{\alpha} \in \mathbb{C}$, and $\left\{\chi_{\alpha}\right\}=\left[\left\{\psi_{s}\right\}\right] \cap \mathcal{O}_{c}$ is the set of primary first class constraints. Following some rigorous work in a presymplectic geometrical framework, Gotay et al. [8] and Gotay [24] concluded that in general the question of whether the secondary first class constraints are to be included in $H_{E}$, i.e. whether they generate gauge transformations or not, is a matter of interpretation. 
There have been various approaches to quantizing the constraint theory of Dirac [2,9-11]. Dirac [2] suggested in the heuristic quantum theory that to quantize degenerate theories, only the reduced phase space functionals are quantized, and this is effected with the rules $\{\cdot, \cdot\}_{D} \rightarrow[\cdot, \cdot] / i \hbar$, and $\alpha_{s}|\psi\rangle=0$, where the latter conditions select the Hilbert states on which the quantum system acts. The second-class constraints therefore do not enter the quantum theory at all. As an alternative one might try to quantize in natural coordinates and in this case the Dirac bracket reverts to the Poisson bracket. Some authors [20] have chosen to interpret the second class constraints in the quantum framework as being algebraic conditions $\xi_{r}=0$. In a later section of this paper we show that this interpretation leads to difficulties, and the best one can do is to quantize in such a way that the second class constraints are absent from the theory from the start. For quantization in a rigorous algebraic framework, it is the suggestion above of Dirac which will be developed here.

Further approaches to quantization of degenerate systems are those of Faddeev [9] who developed path integral quantization for such systems, and Bergman and Goldberg [10] who suggested the following. A classical system can be described by its set of canonical transformations, and these will be unitary transformations in the quantum theory. An invariant subgroup of this group of canonical transformations will be the group of transformations generated by the first class constraints. Then the factor group of the canonical transformations on the system, modulo this invariant subgroup is formed. The new system described by this factor group will be constraint free, and we can quantize as usual. This approach is in the spirit of what we intend to do in the quantum mechanical framework. However in view of the results [8,24], it would seem that we might have to modify the Bergman and Goldberg approach by excluding from the abovementioned subgroup all the first class secondary constraints that do not generate gauge transformations.

Apart from Dirac's canonical constraint theory, there are other ways of dealing with degenerate systems. The traditional way is the use of a supplementary condition. Given an infinite class of solutions, it is possible to select one of them by imposing more conditions viz. a supplementary condition. Whilst there is some freedom in the choice of a supplementary condition, it is clear that it cannot be totally arbitrary as the condition must select one and only one solution. The resulting theory, said to be in a specific "gauge," has highly individual features in its form, some of which might be undesirable. In the quantization of a theory with supplementary conditions, these conditions are chosen to be of only two kinds, algebraic conditions: $\xi_{r}=0$, and state conditions: $\alpha_{s}|\psi\rangle=0$. As examples, consider the different treatments of electromagnetism in $[12,23]$.

Because of the different structure of the quantum system from that of the classical system, there are firstly, more alternatives for dealing with degenerate systems, and secondly, quantum degeneracies which have no classical counterpart. An example of the former is the so-called Fermi trick. This consists of adding an extra term to the Lagrangian so that it is nondegenerate, and then to select only those states on which this extra term will vanish - once more a state condition. In the second instance, the quantum degeneracies are described by gauge transformations. One then eliminates these by imposing either state conditions or algebraic 
conditions on the generators of the gauge transformations (this corresponds to the selection of a "gauge invariant algebra"). No other kind of condition than state or algebraic conditions seems possible.

For a general system, quantization is not a well-defined map from the phase space functionals to the set of Hilbert space operators, since in imposing for instance the requirements $\{\cdot, \cdot\} \rightarrow[\cdot, \cdot] / i \hbar, \mathbb{1}_{\mathrm{Cl}} \rightarrow \mathbb{1}_{\mathrm{QM}}$, one has to contend with Groenewold's theorem [13], which leads to inconsistencies. Following the discussion in [21], it seems that the best one can do in quantizing a general system is to assume a structure in some quantum framework which will more or less mimic the classical structure. The quantum framework that we choose to work in is that of algebraic field theory - the language of $C^{*}$-algebras $[14,15]$. This choice is due to the rich, well-controlled theory available for $C^{*}$-algebras.

In the algebraic framework we will have to assume state and algebraic conditions for dealing with degeneracies, corresponding to the various requirements discussed above. To overcome problems with unboundedness, we can define in the heuristic framework: $\mathbf{U}_{i}(\lambda)=\exp \left(i \lambda \chi_{i}\right), \lambda \in \mathbb{R}$, where all the conditions $\chi_{i}$ can always be chosen to be self adjoint. Then the algebraic conditions are $\mathbf{U}_{r}(\lambda)=\mathbb{1}$, and the state conditions are $\mathbf{U}_{s}(\lambda)|\psi\rangle=|\psi\rangle$. We assume in the $C^{*}$-framework an analogous structure.

\section{Basic Structure and State Conditions}

Following [14], we assume:

Assumption 2.1. $\exists$ a $C^{*}$-algebra $\mathscr{F}$, called the "field algebra," and a set of states $\mathfrak{S}$ on it, and all physical information is contained in this pair. The algebra $\mathscr{F}$ has a unit, $\mathbb{1}$.

Assumption 2.2. $\exists$ two families of one-parameter groups: $\left\{\mathbf{U}_{i}(\lambda) \mid \lambda \in \mathbb{R}, i \in I\right\}$ and $\left\{\mathbf{V}_{i}(\lambda) \mid \lambda \in \mathbb{R}, i \in J\right\}$ in $\mathscr{F}$, where $I$ and $J$ are index sets which need not be finite. Call $\left\{\mathbf{U}_{i}(\lambda)\right\}$ the state conditions, and $\left\{\mathbf{V}_{i}(\lambda)\right\}$ the algebraic conditions.

In what follows, we develop the theory associated with the state conditions without reference to the algebraic conditions, since the theory of these, and the compatibility questions will be considered in a later section.

We need to impose the state conditions in the algebraic framework in a way analogous to $\mathbf{U}_{i}(\lambda)|\psi\rangle=|\psi\rangle \forall i \in I, \lambda \in \mathbb{R}$. Since every state on $\mathscr{F}$ is a vector state in its GNS representation, it seems that the weakest selection condition for a state $\omega \in \mathbb{E}$ would be

$$
\left\langle\omega ; \mathbf{U}_{i}(\lambda)\right\rangle=1 \forall i \in I, \lambda \in \mathbb{R} .
$$

There are other stronger conditions, e.g. $\left\langle\omega ; A \mathbf{U}_{i}(\lambda)\right\rangle=\langle\omega ; A\rangle=\left\langle\omega ; \mathbf{U}_{i}(\lambda) A\right\rangle$ $\forall i \in I, \forall \lambda \in \mathbb{R}, \forall A \in \mathscr{F}$; but we will prove that they are equivalent. Define the Dirac states as the elements of

$$
\mathfrak{S}_{D}=\left\{\omega \in \mathfrak{S} \mid\left\langle\omega ; \mathbf{U}_{i}(\lambda)\right\rangle=1 \forall i \in I, \lambda \in \mathbb{R}\right\} .
$$

If we define

$$
\mathbf{L}_{i}(\lambda)=\mathbf{U}_{i}(\lambda)-\mathbb{1},
$$


and let $\mathscr{A}(\mathbf{L})$ be the $C^{*}$-subalgebra of $\mathscr{F}$ generated by $\left\{\mathbf{L}_{i}(\lambda) \mid \lambda \in \mathbb{R}, i \in I\right\}$, then $\omega \in \mathfrak{S}_{D}$ iff $\mathbf{L}_{i}(\lambda) \in \operatorname{Ker} \omega \forall i \in I, \lambda \in \mathbb{R}$. The stronger condition mentioned above can be written $\left\langle\omega, A \mathbf{L}_{i}(\lambda)\right\rangle=0=\left\langle\omega, \mathbf{L}_{i}(\lambda) A\right\rangle \forall i \in I, \lambda \in \mathbb{R}, A \in \mathscr{F}$. We use the following notation: if $\Omega \subset \mathscr{F}$ is any set, then $[\Omega]$ denotes the closed linear space in $\mathscr{F}$ generated by $\Omega$ (closed in the $C^{*}$-norm).

Theorem 2.6. (i) If $\exists$ an $\omega \in \mathbb{S}$ such that $\left\{\mathbf{L}_{i}(\lambda) \mid i \in I, \lambda \in \mathbb{R}\right\} \subset \operatorname{Ker} \omega$, then $\mathscr{A}(\mathbf{L})$ C $\operatorname{Ker} \omega$.

(ii) Let $\mathscr{A}$ be any sub-C*-algebra of $\mathscr{F}$. If $\exists$ an $\omega \in \mathbb{S}$ such that $\mathscr{A} \subset \operatorname{Ker} \omega$, then $[\mathscr{A} \mathscr{F} \cup \mathscr{F} \mathscr{A}] \subset \operatorname{Ker} \omega$.

Proof. (i) Let $\left\{\mathbf{L}_{i}(\lambda)\right\} \subset \operatorname{Ker} \omega$. Then from (2.5) we get $\mathbf{L}_{i}(\lambda) \mathbf{L}_{i}^{*}(\lambda)=-\mathbf{L}_{i}^{*}(\lambda)-\mathbf{L}_{i}(\lambda)$, and hence $\left\langle\omega ; \mathbf{L}_{i}(\lambda) \mathbf{L}_{i}^{*}(\lambda)\right\rangle=0$. Using the Cauchy Schwarz inequality,

$$
\left|\left\langle\omega ; \mathbf{L}_{i}(\lambda) F\right\rangle\right|^{2} \leqq\left\langle\omega ; \mathbf{L}_{i}(\lambda) \mathbf{L}_{i}^{*}(\lambda)\right\rangle\left\langle\omega ; F^{*} F\right\rangle=0 \forall F \in \mathscr{F},
$$

we get

$$
\left\langle\omega ; \mathbf{L}_{i}(\lambda) F\right\rangle=0 \forall F \in \mathscr{F}, i \in I, \lambda \in \mathbb{R} .
$$

Since $\left\{\mathbf{L}_{i}(\lambda)\right\} \subset \mathscr{F}$, this means that all products $\mathbf{L}_{i}(\lambda) \ldots \mathbf{L}_{j}(\gamma) \subset \operatorname{Ker} \omega$. Then because $\operatorname{Ker} \omega$ is a closed involutive linear space, this means that $\mathscr{A}(\mathbf{L}) \subset \operatorname{Ker} \omega$.

(ii) Let $\mathscr{A} \subset \operatorname{Ker} \omega$, then $\left\langle\omega ; A A^{*}\right\rangle=0 \forall A \in \mathscr{A}$. Thus $\forall F \in \mathscr{F}, A \in \mathscr{A}$ : $|\langle\omega ; A F\rangle|^{2} \leqq\left\langle\omega ; A A^{*}\right\rangle\left\langle\omega ; F^{*} F\right\rangle=0$ or $\mathscr{A} \mathscr{F} \subset \operatorname{Ker} \omega$. Since $\operatorname{Ker} \omega$ is an involutive set, $\mathscr{F} \mathscr{A} \subset \operatorname{Ker} \omega$, and since $\operatorname{Ker} \omega$ is a closed linear space, $[\mathscr{A} \mathscr{F} \cup \mathscr{F} \mathscr{A}]$ $\subset \operatorname{Ker} \omega$.

This theorem shows the equivalence of Condition (2.3), and the stronger condition: $\left\langle\omega ; A \mathbf{U}_{i}(\lambda)\right\rangle=\langle\omega ; A\rangle=\left\langle\omega ; \mathbf{U}_{i}(\lambda) A\right\rangle$. Since Theorem 2.6 says that $\omega \in \mathfrak{S}_{D}$ iff $\mathscr{A}(\mathbf{L}) \subset \operatorname{Ker} \omega$ iff $[\mathscr{A}(\mathbf{L}) \mathscr{F} \cup \mathscr{F} \mathscr{A}(\mathbf{L})] \subset \operatorname{Ker} \omega$, we can look at the existence of Dirac states:

Theorem 2.7. Let $\mathscr{A}$ be any $C^{*}$-subalgebra of $\mathscr{F}$. Then the following conditions are equivalent:

(i) $\exists \omega \in \mathbb{S}$ such that $\mathscr{A} \subset \operatorname{Ker} \omega$;

(ii) $\mathbb{1} \notin[\mathscr{F} \mathscr{A} \cup \mathscr{A} \mathscr{F}]$;

(iii) $\mathbb{1} \notin \mathscr{A}$.

In this case $\omega$ may be chosen to be pure.

Proof. (i) $\Rightarrow$ (ii). If $\exists \omega \in \mathbb{S}$ such that $\mathscr{A} \subset \operatorname{Ker} \omega$, then $[\mathscr{A} \mathscr{F} \cup \mathscr{F} \mathscr{A}] \subset \operatorname{Ker} \omega$. If $\mathbb{1} \in[\mathscr{F} \mathscr{A} \cup \mathscr{A} \mathscr{F}]$, then $\langle\omega ; \mathbb{1}\rangle=0$, which is contradictory with the definition of a state.

(ii) $\Rightarrow$ (i). Let $\mathbb{1} \notin[\mathscr{F} \mathscr{A} \cup \mathscr{A} \mathscr{F}]$. Now $[\mathscr{F} \mathscr{A}]$ is a closed left ideal for $\mathscr{F}$, and is proper because: $[\mathscr{F} \mathscr{A}] \subset[\mathscr{F} \mathscr{A} \cup \mathscr{A} \mathscr{F}]$, and thus $\mathbb{1} \notin[\mathscr{F} \mathscr{A}], \mathbb{1} \in \mathscr{F}$.

By Pedersen, 3.13.5 [17], each closed left ideal $L$ in a $C^{*}$-algebra $\mathscr{F}$ is the intersection of those left kernels of pure states of $\mathscr{F}$ which contain $L$. The left kernel of a state is the set

$$
N_{\omega}=\left\{F \in \mathscr{F} \mid F^{*} F \in \operatorname{Ker} \omega\right\} .
$$

It is also true that $N_{\omega}+N_{\omega}^{*} \cong \operatorname{Ker} \omega$. Thus by this theorem, $\exists \omega \in \mathfrak{S}^{P}$ (superscript $P$ indicates pure states) such that $[\mathscr{F} \mathscr{A}] \subseteq N_{\omega}$, i.e. $\mathscr{A} \subset[\mathscr{F} \mathscr{A}] \subseteq N_{\omega} \subset \operatorname{Ker} \omega$ which also proves the last statement. 
Since trivially (ii) $\Rightarrow$ (iii), we only have to prove (iii) $\Rightarrow$ (ii). Assume $\mathbb{1} \notin \mathscr{A}$. Since $\mathscr{A}$ is a $C^{*}$-algebra, we can define its multiplier algebra:

$$
M(\mathscr{A})=\{F \in \mathscr{F} \mid F A \in \mathscr{A}, A F \in \mathscr{A} \forall A \in \mathscr{A}\},
$$

i.e. the largest set for which $\mathscr{A}$ is a two-sided ideal. A moment's reflection will show that $M(\mathscr{A})$ is a $C^{*}$-algebra, and that $\mathscr{A}$ is a proper subalgebra of $M(\mathscr{A})$ iff $\mathbb{1} \notin \mathscr{A}$.

Therefore, in this case, $\mathscr{A}$ is a proper ideal of $M(\mathscr{A})$, and we see that $\mathbb{1} \notin[\mathscr{A} M(\mathscr{A}) \cup M(\mathscr{A}) \mathscr{A}]=\mathscr{A}$. Then by the first part of this theorem, $\exists$ a state $\omega$ on $M(\mathscr{A})$ such that $\mathscr{A} \subset \operatorname{Ker} \omega$. By the Hahn Banach theorem $\exists$ an extension $\tilde{\omega}$ of $\omega$ to $\mathscr{F}$. Since $\tilde{\omega}\lceil M(\mathscr{A})=\omega, \operatorname{Ker} \omega=\operatorname{Ker} \tilde{\omega} \cap M(\mathscr{A})$, i.e. $\operatorname{Ker} \omega \cong \operatorname{Ker} \tilde{\omega}$, we get $\mathscr{A} \subset \operatorname{Ker} \tilde{\omega}$. Hence by the first part of this theorem, $\mathbb{1} \notin[\mathscr{F} \mathscr{A} \cup \mathscr{A} \mathscr{F}]$.

We have therefore obtained the simple criterion $\mathbb{1} \notin \mathscr{A}(\mathbf{L})$, for Dirac states to exist, and the satisfaction of this criterion depends only on the nature of the state conditions given. If we denote by $\mathfrak{S}_{D}^{P}$ the set of pure states contained in $\mathfrak{S}_{D}$, then Pedersen 3.13.5 quoted above actually implies the stronger statement:

$$
[\mathscr{F} \mathscr{A}(\mathbf{L})]=\bigcap_{\omega \in \mathfrak{G}_{D}^{P}} N_{\omega} .
$$

Henceforth, to ensure nontriviality of the system we assume:

Assumption 2.11. $\mathbb{1} \notin \mathscr{A}(\mathbf{L})$. Thus from arguments above, $\mathscr{N}=[\mathscr{F} \mathscr{A}(\mathbf{L})]$ is a proper closed left ideal of $\mathscr{F}$. We can improve on (2.10):

Theorem 2.12. $\mathcal{N}=\bigcap_{\omega \in \mathfrak{S}_{D}} N_{\omega}$.

Proof. Since $\bigcap_{\omega \in \mathfrak{S}_{D}} N_{\omega} \subseteq \bigcap_{\omega \in \in_{D}^{P}} N_{\omega}$, it would suffice, via (2.10), to show that $\mathscr{N} \leqq N_{\omega} \forall \omega \in \subseteq_{D}$. Let $F \in \mathscr{N}$, then $F^{*} F \in \mathscr{N}$ since $\mathscr{N}$ is a proper left ideal of $\mathscr{F}$. Then

$$
F^{*} F \in \mathscr{N} \subset[\mathscr{F} \mathscr{A}(\mathbf{L}) \cup \mathscr{A}(\mathbf{L}) \mathscr{F}] \subset \operatorname{Ker} \omega \forall \omega \in \Im_{D},
$$

i.e. $F \in N_{\omega}$ by $(2.8)$.

Theorem 2.13. Define $\mathscr{D}=\mathscr{N} \cap \mathcal{N}^{*}=[\mathscr{F} \mathscr{A}(\mathbf{L})] \cap[\mathscr{A}(\mathbf{L}) \mathscr{F}]$. Then $\mathscr{D}$ is the largest $C^{*}$-algebra in $\bigcap_{\omega \in \mathfrak{S}_{D}} \operatorname{Ker} \omega \equiv \mathscr{K}$.

Proof. We first show that $\mathscr{D}$ is a $C^{*}$-algebra. Since $\mathscr{N}$ and $\mathscr{N}^{*}$ are closed linear spaces, so is $\mathscr{D}$, and it is also clear that $\mathscr{D}$ is an involutive set. For products, let $D$, $D_{0} \in \mathscr{D}$. Then $D D_{0} \in \mathscr{F} \mathscr{N} \subset \mathscr{N}$ and $D D_{0} \in \mathscr{N}^{*} \mathscr{F} \subset \mathscr{N}^{*}$, i.e. $D D_{0} \in \mathscr{N} \cap \mathscr{N}^{*}=\mathscr{D}$. Thus $\mathscr{D}$ is a closed ${ }^{*}$-algebra of the $C^{*}$-algebra $\mathscr{F}$, and hence a $C^{*}$-algebra. Now for maximality. Let $\mathscr{M}$ be any $C^{*}$-algebra contained in $\mathscr{K}$, i.e. $\mathscr{M} \subset \operatorname{Ker} \omega \forall \omega \in \mathfrak{S}_{D}$. Since $\mathscr{M}$ is a $C^{*}$-algebra, $K K^{*} \in \mathscr{M} \subset \operatorname{Ker} \omega$ and $K^{*} K \in \mathscr{M} \subset \operatorname{Ker} \omega$ $\forall K \in \mathscr{M} \forall \omega \in \Im_{D}$. Thus $\mathscr{M} \subset N_{\omega} \cap N_{\omega}^{*} \forall \omega \in \Im_{D}$, i.e.

$$
\mathscr{M} \cong \bigcap_{\omega \in \mathfrak{S}_{D}}\left(N_{\omega} \cap N_{\omega}^{*}\right)=\left(\bigcap_{\omega \in \mathfrak{S}_{D}} N_{\omega}\right) \cap\left(\bigcap_{\omega \in \mathfrak{S}_{D}} N_{\omega}^{*}\right)=\mathscr{N} \cap \mathscr{N}^{*}=\mathscr{D},
$$

via Theorem 2.12. Thus $\mathscr{M} \subseteq \mathscr{D} \forall C^{*}$-algebras $\mathscr{M} \subset \mathscr{K}$. 
Thus $\mathscr{D}$ is the largest $C^{*}$-algebra annihilated by the state conditions (2.3). For any set $\Omega \subset \mathscr{F}$, define its "weak commutant" as

$$
\Omega_{w}^{\prime}=\{F \in \mathscr{F} \mid[F, H] \in \Omega \forall H \in \Omega\} .
$$

Then for the commutant, $\Omega^{\prime} \subset \Omega_{w}^{\prime}$ iff $\{0\} \subset \Omega$, and hence since $\{0\} \subset \mathscr{A}(\mathbf{L}) \subset \mathscr{D}$, we have $\mathscr{D}^{\prime} \subset \mathscr{D}_{w}^{\prime}$. In this situation, $\mathscr{D}_{w}^{\prime}$ corresponds roughly to Dirac's concept of "first class variables," which he took as his observables. We will take $\mathscr{D}_{w}^{\prime}$ to be our observables.

Theorem 2.15. $\mathcal{O} \equiv \mathscr{D}_{w}^{\prime}=M(\mathscr{D})$ (cf. (2.9)).

Proof. If $F \in M(\mathscr{D})$, then $F \mathscr{D} \subset \mathscr{D}, \mathscr{D} F \subset \mathscr{D}$, hence $[F, \mathscr{D}] \subset \mathscr{D}$ or $M(\mathscr{D}) \subseteq \mathscr{D}_{w}^{\prime}$. For reverse inclusion, let $F \in \mathscr{D}_{w}^{\prime}, D \in \mathscr{D}$. Then $[F, D]=D_{F} \in \mathscr{D}$, i.e. $F D=D F$ $+D_{F} \in \mathscr{D}_{w}^{\prime} \mathscr{D}$. Now $D \in \mathscr{D}=\mathscr{N} \cap \mathscr{N}^{*}$, implies $\exists$ two Cauchy sequences in $\mathscr{N}$ and $\mathscr{N}^{*}$ respectively, converging to the same element $D$, or in other words:

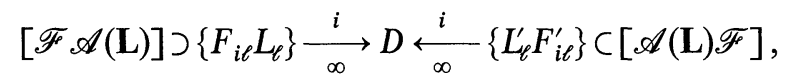

where $L_{\ell}, L_{\ell}^{\prime} \in \mathscr{A}(\mathbf{L}) ; F_{i \ell}, F_{i \ell}^{\prime} \in \mathscr{F}$, and we have finite summation over repeated indices. Also, since $D_{F} \in \mathscr{D}, \exists$ a Cauchy sequence $\left\{L_{\ell}^{\prime \prime} F_{i \ell}^{\prime \prime}\right\} \underset{\infty}{\stackrel{i}{\longrightarrow}} D_{F}$ where $L_{\ell}^{\prime \prime} \in \mathscr{A}(\mathbf{L})$, $F_{i \ell}^{\prime \prime} \in \mathscr{F}$. Hence by continuity of multiplication and addition, we have Cauchy sequences

$$
\left\{F F_{i \ell} L_{\ell}\right\} \underset{\infty}{\stackrel{i}{\longrightarrow}} F D=D F+D_{F} \stackrel{i}{\stackrel{i}{\infty}}\left\{L_{\ell}^{\prime} F_{i \ell}^{\prime} F+L_{\ell}^{\prime \prime} F_{i \ell}^{\prime \prime}\right\} \text {. }
$$

Thus we have two Cauchy sequences, both converging to the same element, with terms contained in $\mathscr{N}$ and $\mathscr{N}^{*}$ respectively. The common limit must therefore be in $\mathscr{D}$, i.e. $F D \in \mathscr{D}$, or $\mathscr{D}_{w}^{\prime} \mathscr{D} \subset \mathscr{D}$. Similarly $\mathscr{D} \mathscr{D}_{w}^{\prime} \subset \mathscr{D}$. Thus $\mathscr{D}$ is a two-sided ideal of $\mathscr{D}_{w}$, i.e. $\mathscr{D}_{w}^{\prime} \subseteq M(\mathscr{D})$.

It is clear from Theorem 2.7 that if $\mathbb{1} \notin \mathscr{A}(\mathbf{L})$, then

$$
\mathbb{1} \notin \mathscr{D}=\mathscr{N} \cap \mathscr{N}^{*} \subset[\mathscr{F} \mathscr{A}(\mathbf{L}) \cup \mathscr{A}(\mathbf{L}) \mathscr{F}] .
$$

Furthermore, $\mathscr{D}$ is a proper closed two-sided ideal of $\mathcal{O}=M(\mathscr{D})$ iff $\mathbb{1} \notin \mathscr{D}$. Thus under Assumption 2.11, $\mathscr{D}$ is proper in $\mathcal{O}$. Since $\mathcal{O}$ is a $C^{*}$-algebra, we can define the $C^{*}$-algebra of the physical observables as

$$
\mathscr{R}:=\mathcal{O} / \mathscr{D} \text {. }
$$

It is clear that $\mathscr{R}$ is a $C^{*}$-algebra with unit, and that it is free of all state conditions $\mathbf{U}_{i}(\lambda)$.

We now present another equivalent algebraic structure which is less intuitive, but perhaps more useful in calculations, and in fact, we will make use of it in a later section.

Let $\mathscr{S} \subset \mathscr{F}$ be the largest set such that

$$
\mathscr{A}(\mathbf{L}) \mathscr{S} \subset[\mathscr{F} \mathscr{A}(\mathbf{L})]
$$

Then $\mathscr{S}^{*} \subset \mathscr{F}$ is the largest set such that $\mathscr{S}^{*} \mathscr{A}(\mathbf{L}) \subset[\mathscr{A}(\mathbf{L}) \mathscr{F}]=\mathscr{N}^{*}$. It is clear that these sets are nonempty because $\mathbb{1} \in \mathscr{A}(\mathbf{L})^{\prime} \subset \mathscr{S} \cap \mathscr{S}^{*}$. 
Theorem 2.18. (i) $\mathscr{D}=\overline{\mathscr{S}^{*} \mathscr{A}(\mathbf{L}) \mathscr{S}}$.

(ii) $\mathcal{O}=\mathscr{S}^{*} \cap \mathscr{S}$, and hence $\mathscr{A}(\mathbf{L})^{\prime} \subset \mathcal{O}$.

Proof. (i) $\overline{\mathscr{S}^{*} \mathscr{A}(\mathbf{L}) \mathscr{S}} \subset \overline{\mathscr{S}^{*}[\tilde{\mathscr{F}} \mathscr{A}(\mathbf{L})]} \subset[\mathscr{F} \mathscr{A}(\mathbf{L})]$ and similarly $\overline{\mathscr{S}^{*} \mathscr{A}(\mathbf{L}) \mathscr{S}} \subset \mathscr{N}^{*}$. Thus $\overline{\mathscr{S}^{*} \mathscr{A}(\mathbf{L}) \mathscr{S}} \subset \mathscr{N} \cap \mathscr{N}^{*}=\mathscr{D}$. From definitions $\mathscr{D} \subset \mathscr{S} \cap \mathscr{S}^{*}$.

Hence: $\mathscr{D}_{0} \equiv \overline{\mathscr{S}^{*} \mathscr{A}(\mathbf{L}) \mathscr{S}} \subset \mathscr{D} \subset \mathscr{S} \cap \mathscr{S}^{*}$. It is not difficult to see that $\mathscr{S}$ and $\mathscr{S}^{*}$ are norm closed algebras, that $\mathscr{S}^{*} \cap \mathscr{S}$ is a $C^{*}$-algebra and that $\mathscr{D}_{0} \equiv \overline{\mathscr{S}^{*} \mathscr{A}(\mathbf{L}) \mathscr{S}}$ is a closed two-sided ideal for it. We prove that it is proper. Since $\mathscr{D}_{0} \subset \mathscr{D} \subset \operatorname{Ker} \omega$ $\forall \omega \in \mathfrak{S}_{D} \neq \emptyset$, we have that $\mathbb{1} \notin \mathscr{D}_{0}$. But $\mathbb{1} \in \mathscr{S} \cap \mathscr{S}^{*}$ and hence $\mathscr{D}_{0}$ is a proper closed two-sided ideal of $\mathscr{S} \cap \mathscr{S}^{*}$. From definitions, $\mathscr{D} \subset \mathscr{S} \cap \mathscr{S}^{*}$, and $\mathscr{D}$ is a twosided ideal of $\mathscr{S} \cap \mathscr{S}^{*}$ because $\left(\mathscr{S} \cap \mathscr{S}^{*}\right) \mathscr{N} \subset \mathscr{N},\left(\mathscr{S} \cap \mathscr{S}^{*}\right) \mathscr{N}^{*} \subset \mathscr{N}^{*}$ via (2.17), and hence $\left(\mathscr{S} \cap \mathscr{S}^{*}\right) \mathscr{D} \subset \mathscr{D}$. Since $\mathscr{S} \cap \mathscr{S}^{*}$ and $\mathscr{D}$ are involutive, $\mathscr{D}$ is a two-sided ideal for $\mathscr{S}_{\cap} \mathscr{S}^{*}$, i.e. $\mathscr{S} \cap \mathscr{S}^{*} \subset M(\mathscr{D})=\mathcal{O}$. Via Dixmier 2.9.4 [18]; if $\mathscr{L}_{1} \subset \mathscr{L}_{2}$ are two closed left ideals of a $C^{*}$-algebra, and every positive functional that vanishes on $\mathscr{L}_{1}$ also vanishes on $\mathscr{L}_{2}$, then $\mathscr{L}_{1}=\mathscr{L}_{2}$. Thus we have to show that $\mathfrak{S}_{\mathscr{D}}\left(\mathcal{O}_{0}\right)$ $=\mathfrak{S}_{\mathscr{D}}\left(\mathcal{O}_{0}\right)$, where $\mathfrak{S}_{\mathscr{D}}\left(\mathcal{O}_{0}\right)$ is the set of states on $\mathcal{O}_{0} \equiv \mathscr{S} \cap \mathscr{S}^{*}$ such that $\mathscr{D} \subset \operatorname{Ker} \omega$, and $\mathfrak{S}_{\mathscr{D}_{0}}\left(\mathcal{O}_{0}\right)$ is the set of states on $\mathcal{O}_{0}$ such that $\mathscr{D}_{0} \subset \operatorname{Ker} \omega$. By the Hahn-Banach theorem, every state on $\mathcal{O}_{0}$ is the restriction of a state on $\mathscr{F}$. Thus since $\operatorname{Ker}\left(\omega \uparrow \mathcal{O}_{0}\right)=\operatorname{Ker} \omega \cap \mathcal{O}_{0}$, we have

$$
\mathscr{D}_{0} \subset \operatorname{Ker}\left(\omega\left\lceil\mathcal{O}_{0}\right) \Rightarrow \mathscr{D}_{0} \subset \operatorname{Ker} \omega \Rightarrow \omega \in \mathbb{S}_{D},\right.
$$

since $\mathscr{A}(\mathbf{L}) \subset \mathscr{D}_{0}$. Then by Theorem 2.13, $\mathscr{D} \subset \operatorname{Ker} \omega \Rightarrow \mathscr{D} \cap \mathscr{O}_{0}=\mathscr{D} \subset \operatorname{Ker}\left(\omega\left\lceil\mathcal{O}_{0}\right)\right.$. Thus every state on $\mathcal{O}_{0}$ that vanishes on $\mathscr{D}_{0}$ vanishes on $\mathscr{D}$, and since every positive functional can be obtained from a state by multiplication by a constant, $\mathscr{D}=\mathscr{D}_{0}$.

(ii) It has been proven above that $\mathcal{O}_{0} \subseteq \mathcal{O}$. Conversely, let $F \in \mathcal{O}$. Then $F \mathscr{D} \subset \mathscr{D}$ and $\mathscr{D} F \subset \mathscr{D}$, i.e. $\mathscr{D} F \subset \mathscr{D} \subset \mathscr{N}$. Since $\mathscr{A}(\mathbf{L}) \subset \mathscr{D}$, this implies $\mathscr{A}(\mathbf{L}) F \subset \mathscr{N} \Rightarrow F \in \mathscr{S}$ by (2.17). Similarly $F \in \mathscr{S}^{*}$ and hence $F \in \mathscr{S} \cap \mathscr{S}^{*}=\mathcal{O}_{0}$. Thus $\mathscr{O} \subseteq \mathcal{O}_{0}$.

Since $\mathscr{A}(\mathbf{L})^{\prime}$ are the traditional gauge invariant observables, the fact that $\mathscr{A}(\mathbf{L})^{\prime} \subset \mathcal{O}$ partly justifies the choice of $\mathcal{O}$ as the set of observables. After exploring these basic algebraic structures associated with conditions (2.3), we now turn our attention to the states on them.

Theorem 2.19. (i) $\mathfrak{S}_{D}$ is convex, closed in the $w^{*}$-topology, and compact. $\mathfrak{S}_{D}$ is the $w^{*}$ closure of the convex hull of its extreme points.

(ii) $\omega \in \mathfrak{S}_{D}$ iff $\pi_{\omega}(D) \Omega_{\omega}=0 \forall D \in \mathscr{D}$, where $\pi_{\omega}, \Omega_{\omega}$ are the GNS representation and associated cyclic state respectively associated with $\omega$.

(iii) Let $\mathfrak{v}_{\omega}$ denote the set of vector states associated with a GNS representation $\pi_{\omega}$, i.e.

$$
\mathfrak{v}_{\omega}=\left\{\omega_{\xi} \in \mathbb{S} \mid\left\langle\omega_{\xi} ; A\right\rangle=\left(\xi ; \pi_{\omega}(A) \xi\right) \forall A \in \mathscr{F} \text { and some } \xi \in \mathscr{H}_{\omega}\right\} \text {. }
$$

Let $\omega \in \Im_{D}$, $\xi \in \mathscr{H}_{\omega}$. Then $\pi_{\omega}(\mathscr{D}) \xi=0$ iff $\omega_{\xi} \in \Im_{D}$.

Proof. (i) Let

$$
\omega=\sum_{i=1}^{n} \lambda_{i} \omega_{i}, \quad \sum_{i=1}^{n} \lambda_{i}=1, \quad \lambda_{i}>0, \quad \omega_{i} \in \mathfrak{S}_{D}
$$

be any finite convex combination of elements of $\mathfrak{S}_{D}$. Since $\mathfrak{S}$ is convex, $\omega \in \mathbb{G}$, and $\lambda_{i}>0$, we have that $\mathscr{A}(\mathbf{L}) \subseteq \operatorname{Ker} \omega=\bigcap_{i=1}^{n} \operatorname{Ker} \omega_{i}, \omega_{i} \in \mathfrak{S}_{D}$. Thus $\omega \in \mathfrak{S}_{D}$, and $\mathfrak{S}_{D}$ is a convex set. Let $\mathscr{A}(\mathbf{L})^{\perp} \subset \mathscr{F}^{*}$ be the annihilator of $\mathscr{A}(\mathbf{L})$ in the dual $\mathscr{F}^{*}$ of $\mathscr{F}$, and 
this is a $w^{*}$-closed subspace of $\mathscr{F}^{*}$ (see Naimark p. 65 [19]). Furthermore $\mathfrak{S} \subset \mathscr{F} *$ is $w^{*}$-closed if $\mathbb{1} \in \mathscr{F}$, and hence $\mathfrak{S}_{D}=\boldsymbol{S}_{\cap} \mathscr{A}(\mathbf{L})^{\perp}$ is $w^{*}$-closed. By Alaoglu's theorem, the closed unit ball in $\mathscr{F}^{*}, \mathfrak{B} .=\left\{\varphi \in \mathscr{F}^{*} \mid\|\varphi\| \leqq 1\right\}$ is compact in the $w^{*}$ topology. But $\mathfrak{S}_{D} \subset \mathfrak{S} \subset \mathfrak{B}$, and any closed subset of a compact set is compact, thus $\mathfrak{S}_{D}$ is $w^{*}$-compact. Furthermore, since $\mathscr{F}^{*}$ is a locally convex linear topological space, the Krein-Milman theorem applies, so that $\mathfrak{S}_{D}$ is the smallest $w^{*}$-closed convex set containing all its extreme points:

$$
\Im_{D}=\operatorname{co}\left(\Im_{D}^{*}\right)
$$

(ii) Since $[\mathscr{F} \mathscr{A}(\mathbf{L})] \subseteq[\mathscr{F} \mathscr{D}] \subseteq[\mathscr{F}[\mathscr{F} \mathscr{A}(\mathbf{L})]] \subseteq[\mathscr{F} \mathscr{A}(\mathbf{L})]$, we have that $[\mathscr{F} \mathscr{A}(\mathbf{L})]=[\mathscr{F} \mathscr{D}]$. Then using Theorem 2.6, $\omega \in \mathfrak{S}_{D}$ iff

$$
0=\langle\omega, F D\rangle=\left(\Omega_{\omega}, \pi_{\omega}(F D) \Omega_{\omega}\right)=\left(\pi_{\omega}\left(F^{*}\right) \Omega_{\omega}, \pi_{\omega}(D) \Omega_{\omega}\right) \forall F \in \mathscr{F}, D \in \mathscr{D} .
$$

Since $\Omega_{\omega}$ is cyclic for $\pi_{\omega}(\mathscr{F})$, we get $\pi_{\omega}(D) \Omega_{\omega}=0 \forall D \in \mathscr{D}$.

(iii) Clearly $\pi_{\omega}(\mathscr{D}) \xi=0 \Rightarrow \omega_{\xi} \in \mathfrak{S}_{D}$. Conversely, if $\omega_{\xi} \in \mathfrak{S}_{D}$, then $\forall D \in \mathscr{D}$, $\left\langle\omega_{\xi}, D^{*} D\right\rangle=\left\|\pi_{\omega}(D) \xi\right\|^{2}=0$.

By Theorem 2.7, $\mathfrak{S}_{D}^{P} \neq \emptyset$, and since $\mathfrak{S}_{D}^{P} \subseteq \mathfrak{S}_{D}^{E}$ (extreme Dirac states), we get that at least some of the extreme Dirac states are pure. The question naturally arises whether they all are. This can be proven in the particular case when $\mathfrak{S}$ is metrizable, e.g. when $\mathscr{F}$ is separable, but not in the general case. The problem is that in the barycentric decomposition of a state $\omega$, the maximal measure on $\subseteq$ corresponding to $\omega$ is only pseudo-supported by the pure states, but not always supported by them [15]. From $[\mathscr{F} \mathscr{A}(\mathbf{L})]=[\mathscr{F} \mathscr{D}]$, proven above, we see that $[\mathscr{F} \mathscr{D}] \cap[\mathscr{D} \mathscr{F}]=\mathscr{D}$, and therefore the definition of $\mathscr{D}$ is consistent, i.e. $\mathscr{D}$ cannot in turn serve as a new constraint algebra $\mathscr{A}(\mathbf{L})$ from which an even larger $\mathscr{D}$ can be constructed. This is also seen from Theorem 2.13 . We can equally define $\mathfrak{S}_{D}$ as the set of states with $\mathscr{D}$ in their kernels.

Theorem 2.20. (i) $\exists a w^{*}$-continuous, isometric bijection between the Dirac states on $\mathcal{O}$, denoted $\mathfrak{\Xi}_{D}(\mathcal{O})$, and the states on $\mathscr{R}, \mathfrak{S}(\mathscr{R})$.

(ii) $\exists$ a bijection between $\mathfrak{S}_{D}^{P}(\mathcal{O})$ and $\mathfrak{S}^{P}(\mathscr{R})$.

(iii) If $\omega \in \Im_{D}(\mathcal{O})$, then $\mathscr{D} \cong \operatorname{Ker} \pi_{\omega}$, so that trivially $\pi_{\omega}(D) \xi=0 \forall D \in \mathscr{D}, \forall \xi \in \mathscr{H}_{\omega}$, Dirac's requirement.

Proof. (i) This follows from $\mathfrak{S}_{D}(\mathcal{O})=\{\omega \in \mathfrak{S}(\mathcal{O}) \mid \mathscr{D} \subseteq \operatorname{Ker} \omega\}$ and Dixmier 2.11.6 [18].

(ii) This follows from Dixmier 2.11.8 [18].

(iii) By Dixmier 2.4.10 [18], Ker $\pi_{\omega}$ is the largest closed two-sided ideal in $\operatorname{Ker} \omega$. But $\mathscr{D} \subset \operatorname{Ker} \omega$ is a closed two-sided ideal of $\mathcal{O}$. Thus $\mathscr{D} \subseteq \operatorname{Ker} \pi_{\omega}$.

By Dixmier 2.10.1 [18], every pure state on $\mathcal{O}$, can be extended to a pure state on $\mathscr{F}$. Hence since $\mathscr{D} \subset \mathcal{O}$, and thus $\mathfrak{S}_{D} \uparrow \mathcal{O}=\mathfrak{S}_{D}(\mathcal{O})$, we get that $\mathfrak{S}_{D}^{P}(\mathcal{O})$ corresponds to a subset of $\mathfrak{S}_{D}^{P}$, i.e. $\omega \in \mathfrak{S}_{D}^{P}(\mathcal{O}) \Rightarrow \exists \tilde{\omega} \in \mathfrak{S}_{D}^{P}$ such that $\tilde{\omega}\lceil\mathcal{O}=\omega$. This gives a partial answer to the significance of the extreme states of $\mathfrak{S}_{D}$ that are not pure states, if they exist. Theorem 2.20 (iii) shows that the constraint condition is an algebraic condition in each GNS representation $\pi_{\omega}$ of a state $\omega \in \mathbb{S}_{D}(\mathcal{O})$. Since $\mathcal{O}$ is a $C^{*-}$ 
algebra, its set of Dirac states will be the $w^{*}$-convex hull of its extreme Dirac states by an argument similar to Theorem 2.19 (i).

Assumption 2.21. All the physical information of the system $\left\langle\mathscr{F}, \mathfrak{S},\left\{\mathbf{U}_{i}(\lambda)\right\}\right\rangle$ is contained in the subsystem $\langle\mathscr{R}, \Im(\mathscr{R})\rangle$.

\section{Transformations}

In this section the compatibility of the structure defined above with various automorphisms of $\mathscr{F}$ is examined. Let $G$ denote the locally compact symmetry group of $\mathscr{F}$. Then we have the triple $\langle\mathscr{F}, G, \alpha\rangle$, where the action $\alpha: G \rightarrow$ Aut $\mathscr{F}$ is a continuous homomorphism. Furthermore, since $\mathscr{F}$ is a $C^{*}$-algebra, each $\alpha_{g} \in$ Aut $\mathscr{F}$ is continuous for $g \in G$ fixed. Call the procedure developed in Sect. 2 for passing from $\left\langle\mathscr{F},\left\{\mathbf{U}_{i}(\lambda)\right\}, \subseteq\right)$ to $\langle\mathscr{R}, \Im(\mathscr{R})\rangle$, the $T$-procedure. Then we would like to determine whether the diagram:

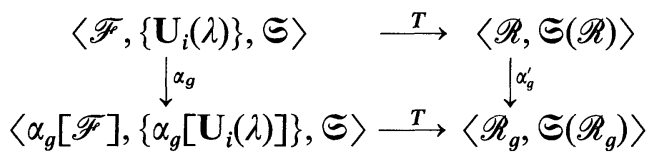

defines a map $\alpha_{g}^{\prime}$ under which it is commutative, and what the properties of $\alpha_{g}^{\prime}$ are. Then we can select a class of transformations for which $\mathscr{R}=\mathscr{R}_{g}$ (equality is in terms of equivalence classes in $\mathscr{F}$, not isomorphism), which we will regard as the physical transformations of the system.

For $\alpha_{g} \in$ Aut $\mathscr{F}$, let $\mathscr{A}_{g}(\mathbf{L})$ be the $C^{*}$-algebra generated by the set $\left\{\alpha_{g}\left[\mathbf{L}_{i}(\lambda)\right] \mid i \in I, \lambda \in \mathbb{R}\right\}$, and $\mathfrak{S}_{D_{g}}, \mathscr{S}_{g}, \mathcal{O}_{g}, \mathscr{D}_{g}$ are the corresponding entities defined by $\mathscr{A}_{g}(\mathbf{L})$ as in Sect. 2 .

Theorem 3.1. (i) $\alpha_{g}[\mathscr{A}(\mathbf{L})]=\mathscr{A}_{g}(\mathbf{L})$.

(ii) $\alpha_{g}[\mathscr{S}]=\mathscr{S}_{g}, \alpha_{g}[\mathcal{O}]=\mathcal{O}_{g}, \alpha_{g}[\mathscr{D}]=\mathscr{D}_{g}$, and $\mathscr{D}_{g}$ is a proper closed two-sided ideal for $\mathscr{O}_{g}$. Thus define $\mathscr{R}_{g}=\mathcal{O}_{g} / \mathscr{D}_{g}$.

(iii) $\exists$ a natural isomorphism between $\mathscr{R}$ and $\mathscr{R}_{g}$, defined by $\alpha_{g}[\xi(A)]=\xi_{g}\left(\alpha_{g}[A]\right)$, where $\xi(A)$ and $\xi_{g}\left(A^{\prime}\right)$ are respectively the equivalence classes $\xi(A)=\{A+D \mid D \in \mathscr{D}\}$, $A \in \mathcal{O}$, and $\xi_{g}(A) .=\left\{A^{\prime}+D^{\prime} \mid D^{\prime} \in \mathscr{D}_{g}\right\}, A^{\prime} \in \mathcal{O}_{g}$. Call this isomorphism $\alpha_{g}^{\prime} ; \mathscr{R} \rightarrow \mathscr{R}_{g}$.

Proof. (i) This follows trivially as $\alpha_{g} \in$ Aut $\mathscr{F}$ is a homomorphism, and thus preserves multiplication, summation, scalar multiplication, involution, and is continuous, so preserves limits.

(ii) Let $S \in \mathscr{S}$. Then $\alpha_{g}[S] \in \alpha_{g}[\mathscr{S}]$, and $\mathscr{A}_{g}(\mathbf{L}) \alpha_{g}[S]=\alpha_{g}[\mathscr{A}(\mathbf{L}) S] \subset \alpha_{g}[\mathscr{N}]$ $=\left[\mathscr{F}_{\mathscr{A}}(\mathbf{L})\right]$. Thus $\alpha_{g}[\mathscr{S}] \subseteq \mathscr{S}_{g}$. Conversely, let $S_{g} \in \mathscr{S}_{g}$. Then $\mathscr{A}_{g}(\mathbf{L}) S_{g}$ $=\alpha_{g}[\mathscr{A}(\mathbf{L})] S_{g} \subset\left[\mathscr{F} \mathscr{A}_{g}(\mathbf{L})\right]=\alpha_{g}[\mathscr{N}]$. Since $G$ is a group, $g^{-1} \exists$, and $\mathscr{A}(L) \alpha_{g}^{-1}\left[S_{g}\right]$ $\subset \mathscr{N}$, i.e. $\alpha_{g}^{-1}\left[S_{g}\right] \in \mathscr{S}$, or $S_{g} \in \alpha_{g}[\mathscr{S}]$. Thus $\mathscr{S}_{g} \subseteq \alpha_{g}[\mathscr{S}]$. Taking adjoints of $\mathscr{S}_{g}$ $=\alpha_{g}[\mathscr{S}]$ gives $\mathscr{S}_{g}^{*}=\alpha_{g}\left[\mathscr{S}^{*}\right]$. Thus $\mathscr{O}_{g}=\mathscr{S}_{g} \cap \mathscr{S}_{g}^{*}=\alpha_{g}[\mathscr{S}] \cap \alpha_{g}\left[\mathscr{S}^{*}\right]=\alpha_{g}\left[\mathscr{S} \cap \mathscr{S}^{*}\right]$ $=\alpha_{g}[\mathcal{O}]$. Furthermore, $\mathscr{D}_{g}=\overline{\mathscr{S}_{g}^{*} \mathscr{A}_{g}(\mathbf{L}) \mathscr{S}_{g}}=\alpha_{g}\left[\mathscr{S}^{*} \mathscr{A}(\mathbf{L}) \mathscr{S}\right]=\alpha_{g}[\mathscr{D}]$. Since $\alpha_{g}$ is a homomorphism, and $\mathscr{D}$ is a closed ideal for $\mathscr{O}, \alpha_{g}[\mathscr{D}]$ will be a closed ideal of $\alpha_{g}[\mathcal{O}]$. Now $\alpha_{g}[\mathbb{1}]=\mathbb{1}$, and since $\alpha_{g}$ is an automorphism, there are no other elements $A \neq \mathbb{1}$ such that $\alpha_{g}[A]=\mathbb{1}$. Thus $\mathbb{1} \notin \mathscr{A}(\mathbf{L}) \Leftrightarrow \mathbb{1} \notin \mathscr{A}_{g}(\mathbf{L})$. Thus $\mathscr{D}_{g}$ is proper in $\mathcal{O}_{g}$.

(iii) $\alpha_{g}[\xi(A)]=\alpha_{g}\left[\left\{A+D \mid D \in \mathscr{D}^{\prime}\right\}\right]=\left\{\alpha_{g}[A]+D_{g} \mid D_{g} \in \mathscr{D}_{g}\right\}=\xi_{g}\left(\alpha_{g}[A]\right)$. We wish to show that this is an isomorphism between $\mathscr{R}$ and $\mathscr{R}_{g}$. It is trivial that 
$\alpha_{g}[\lambda \xi(A)]=\lambda \alpha_{g}[\xi(A)], \quad \lambda \in \mathbb{C}, \quad$ and $\quad \alpha_{g}[\xi(A)+\xi(B)]=\alpha_{g}[\xi(A)]+\alpha_{g}[\xi(B)] \quad$ and $\alpha_{g}[\xi(A)]^{*}=\alpha_{g}\left[\xi\left(A^{*}\right)\right]$. For products

$$
\begin{aligned}
\alpha_{g}[\xi(A) \xi(B)] & =\alpha_{g}\left[\left\{(A+D)\left(B+D^{\prime}\right) \mid D, D^{\prime} \in \mathscr{D}\right\}\right] \\
& =\left\{\left(\alpha_{g}[A]+D_{g}\right)\left(\alpha_{g}[B]+D_{g}^{\prime}\right) \mid D_{g}, D_{g}^{\prime} \in \mathscr{D}_{g}\right\} \\
& =\xi_{g}\left(\alpha_{g}[A]\right) \xi_{g}\left(\alpha_{g}[B]\right)=\alpha_{g}[\xi(A)] \alpha_{g}[\xi(B)] .
\end{aligned}
$$

Thus $\alpha_{g}^{\prime}$ is a homomorphism. The zero equivalence class in $\mathscr{R}$ (respectively $\mathscr{R}_{g}$ ) is $\mathscr{D}$ (respectively $\mathscr{D}_{g}$ ). Now $\alpha_{g} \in$ Aut $\mathscr{F} \Rightarrow \operatorname{Ker} \alpha_{g}=\{0\}$, and this still holds for $\alpha_{g} \uparrow \mathcal{O}$, i.e. $\alpha_{g}$ is an isomorphism from $\mathcal{O}$ to $\alpha_{g}[\mathcal{O}]$. By the form of the defining equality for $\alpha_{g}^{\prime}$, we have $\operatorname{Ker} \alpha_{g}^{\prime}=\alpha_{g}^{-1}\left[\mathscr{D}_{g}\right]$, i.e. $\xi(A) \in \operatorname{Ker} \alpha_{g}^{\prime}$ if $\alpha_{g}[\xi(A)]=\mathscr{D}_{g}=\xi_{g}(0)=\xi_{g}\left(\alpha_{g}[A]\right)$. Then $\alpha_{g}[A] \in \mathscr{D}_{g}$, or $A \in \alpha_{g}^{-1}\left[\mathscr{D}_{g}\right]=\alpha_{g}^{-1}\left[\alpha_{g}[\mathscr{D}]\right]=\mathscr{D}$. Thus $\operatorname{Ker} \alpha_{g}^{\prime}=\mathscr{D}=\xi(0)$ and $\alpha_{g}^{\prime}$ is an isomorphism.

Whilst $\mathscr{R}$ and $\mathscr{R}_{g}$ are isomorphic, we really have no way of visualizing $\alpha_{g}$ as a physical transformation, unless there is some "identity" isomorphism between $\mathscr{R}$ and $\mathscr{R}_{g}$ to compare $\alpha_{g}$ with. For this reason we wish to restrict ourselves to those $\alpha_{g} \in$ Aut $\mathscr{F}$ such that $\mathscr{R}=\mathscr{R}_{g}$ in the sense of equivalence classes in $\mathcal{O}$. In this case $\mathscr{O}=\mathcal{O}_{g}, \mathscr{D}=\mathscr{D}_{g}$. However since $\mathcal{O}=M(\mathscr{D})$, the requirement $\mathscr{D}=\mathscr{D}_{g}$ suffices to guarantee that $\mathscr{R}=\mathscr{R}_{g}$. Define

$$
\mathfrak{P} .=\left\{\alpha_{g} \in \text { Aut } \mathscr{F} \mid g \in G, \mathscr{D}=\mathscr{D}_{g}\right\} .
$$

Theorem 3.1 says that the $T$-procedure maps $\left\{\alpha_{g} \mid g \in G\right\}$ into $\operatorname{Isom}(\mathscr{R}, \mathscr{R} g)$, and specifically maps $\mathfrak{P}$ into Aut $\mathscr{R}$. Symbolically, define $T ; \mathfrak{P} \rightarrow$ Aut $\mathscr{R}$ by $T\left(\alpha_{g}\right)=\alpha_{g}^{\prime} \in$ Aut $\mathscr{R}, \alpha_{g} \in \mathfrak{P}$.

Theorem 3.3. (i) $\mathfrak{P}$ is a group.

(ii) $T: \mathfrak{P} \rightarrow$ Aut $\mathscr{R}$ is a homomorphism, and thus $\mathfrak{P} / \operatorname{Ker} T$ is isomorphic to $T(\mathfrak{P})$ CAut $\mathscr{R}$.

(iii) $\alpha_{g} \in \operatorname{Ker} T$ iff $\alpha_{g}[A]=A+D_{g}(A) \forall A \in \mathcal{O}$, where $D_{g}: \mathcal{O} \rightarrow \mathscr{D}$ is an involutive linear map satisfying $\forall A, B \in \mathcal{O}$ :

$$
D_{g}(A B)=A D_{g}(B)+D_{g}(A) B+D_{g}(A) D_{g}(B) .
$$

In this case $D_{g}(\mathbb{1})=0$.

Proof. (i) It is clear from (3.2) that the successive application of elements in $\mathfrak{P}$ yields an element of $\mathfrak{P}$, and that the identity $\alpha_{e} \in \mathfrak{P}$, where $e$ is the identity of $G$.

If $\alpha_{g} \in \mathfrak{P}$, then $\alpha_{g}^{-1} \in \mathfrak{P}$, since $\mathscr{D}=\mathscr{D}_{g}=\alpha_{g}[\mathscr{D}] \Leftrightarrow \alpha_{g}^{-1}[\mathscr{D}]=\mathscr{D}$.

(ii) Let $\alpha_{g}, \alpha_{h} \in \mathfrak{B}$. Then $\forall \xi(A) \in \mathscr{R}, A \in \mathcal{O}$,

$$
\begin{aligned}
T\left(\alpha_{g} \alpha_{h}\right)[\xi(A)] & =\alpha_{g h}[\{A+D \mid D \in \mathscr{D}\}]=\left\{\alpha_{g h}[A]+\alpha_{g h}[D] \mid D \in \mathscr{D}\right\} \\
& =\left\{\alpha_{g h}[A]+D \mid D \in \mathscr{D}\right\}=\xi\left(\alpha_{g} \alpha_{h}[A]\right)
\end{aligned}
$$

and

$$
T\left(\alpha_{g}\right) T\left(\alpha_{h}\right)[\xi(A)]=T\left(\alpha_{g}\right)\left[\xi\left(\alpha_{h}[A]\right)\right]=\xi\left(\alpha_{g} \alpha_{h}[A]\right)=T\left(\alpha_{g} \alpha_{h}\right)[\xi(A)] .
$$

(iii) $\alpha_{g} \in \operatorname{Ker} T$ iff $T\left(\alpha_{g}\right)=\mathbb{1}_{\text {Aut } \mathscr{R}}$, i.e.

$$
T\left(\alpha_{g}\right)[\xi(A)]=\xi(A) \forall A \in \mathcal{O},
$$


or

$$
\begin{aligned}
T\left(\alpha_{g}\right)[\xi(A)] & =\xi\left(\alpha_{g}[A]\right)=\left\{\alpha_{g}[A]+D \mid D \in \mathscr{D}\right\} \\
& =\xi(A)=\{A+D \mid D \in \mathscr{D}\} \forall A \in \mathcal{O},
\end{aligned}
$$

and thus $\alpha_{g}[A]=A+D_{g}(A)$, where $D_{g}(A) \in \mathscr{D} \forall A \in \mathcal{O}$. Since $\alpha_{g}$ is linear and involutive we get that $D_{g}: \mathcal{O} \rightarrow \mathscr{D}$ is linear and involutive. Furthermore we find

$$
\begin{aligned}
\forall A, B \in \mathcal{O}: \alpha_{g}[A B] & =A B+D_{g}(A B)=\alpha_{g}[A] \alpha_{g}[B] \\
& =A B+A D_{g}(B)+D_{g}(A) B+D_{g}(A) D_{g}(B),
\end{aligned}
$$

and (3.4) is clear. Now since $\alpha_{g}$ is an automorphism, we have $\alpha_{g}[\mathbb{1}]=\mathbb{1}$. But $\alpha_{g}[A]$ $=A+D_{g}(A)$. Thus $D_{g}(\mathbb{1})=0$.

It is clear that if $\mathscr{A}_{g}(\mathbf{L})=\mathscr{A}(\mathbf{L})$, then $\alpha_{g} \in \mathfrak{P}$, via defining relations. The converse will not hold in general, since if $\alpha_{g}[\mathscr{A}(\mathbf{L})]=\mathscr{D}$, then we still get that $\alpha_{g} \in \mathfrak{P}$, and we do not have generally that $\mathscr{A}(\mathbf{L})=\mathscr{D}$. Note that it is only the behaviour of an $\alpha_{g} \in$ Aut $\mathscr{F}$ on $\mathcal{O}$, which matters in determining whether it is in $\mathfrak{P}$ or Ker T. Outside of $\mathcal{O}$, its behaviour can be arbitrary. If $\alpha_{g} \in \mathfrak{P}$, then since $\mathscr{D}=\mathscr{D}_{g}$, we have that $\Im_{D_{g}}$

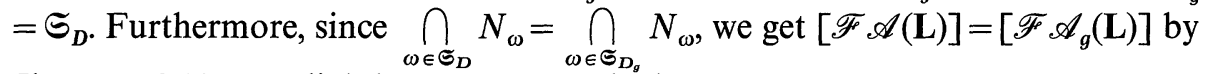
Theorem 2.12 ; - a slightly stronger result than $\mathscr{D}=\mathscr{D}_{g}$.

We would now like to determine the nature of the transformations eliminated from the system in going from $\mathscr{F}$ to $\mathscr{R}$, i.e. the transformations in $\mathrm{Ker} T$. This group of transformations should contain all transformations generated by the constraints $\left\{\mathbf{U}_{i}(\lambda)\right\}$, and indeed all those generated by $\mathscr{D}$. Then the question arises as to what other transformations are eliminated from the theory, and whether they are physical or not. Define the following groups of gauge transformations:

$$
\begin{gathered}
\mathscr{G}(\chi)=\left\{\alpha_{i, \lambda} \in \text { Aut } \mathscr{F} \mid \alpha_{i, \lambda}[A] .=\mathbf{U}_{i}(\lambda) A \mathbf{U}_{i}(\lambda)^{-1} \forall A \in \mathscr{F}, \lambda \in \mathbb{R}\right\}, \\
\mathscr{G}_{u}(\mathscr{D}) .=\left\{\alpha_{D, \lambda} \in \operatorname{Aut} \mathscr{F} \mid \alpha_{D, \lambda}[A]\right. \\
\left..=\exp (i \lambda D) A \exp (-i \lambda D), A \in \mathscr{F}, \lambda \in \mathbb{R}, D \in \mathscr{D}_{h}\right\}, \\
\mathscr{G}(\mathscr{D})=\left\{\alpha_{D} \in \text { Aut } \mathscr{F} \mid \alpha_{D}[A] .=(\mathbb{1}+D) A\left(\mathbb{1}+D^{*}\right)\right. \\
\left.\forall A \in \mathscr{F} \text { and } \forall D \in \mathscr{D} \text { such that } D+D^{*}=-D D^{*}=-D^{*} D\right\} .
\end{gathered}
$$

The last condition in (3.6) ensures that $(\mathbb{1}+D)$ is unitary:

$$
(\mathbb{1}+D)\left(\mathbb{1}+D^{*}\right)=\mathbb{1}=\left(\mathbb{1}+D^{*}\right)(\mathbb{1}+D) .
$$

It is easy to see that all these transformations are gauge transformations, because they leave Dirac state expectation values unchanged:

$$
\left\langle\omega, \alpha_{i, \lambda}[A]\right\rangle=\left\langle\omega, \alpha_{D, \lambda}[A]\right\rangle=\left\langle\omega, \alpha_{D}[A]\right\rangle=\langle\omega, A\rangle \forall \omega \in \mathfrak{S}_{D}, A \in \mathscr{F} .
$$

It is not clear that $\mathscr{G}(\mathscr{D})$ is a group.

Theorem 3.7. (i) $\mathscr{G}(\mathscr{D})$ is a group, and $\mathscr{G}_{u}(\mathscr{D}) \subset \mathscr{G}(\mathscr{D})$;

(ii) $\mathscr{G}(\chi) \subset \mathscr{G}(\mathscr{D}) \subseteq \operatorname{Ker} T$.

Proof. (i) We first show that $\mathscr{G}(\mathscr{D})$ is a group. Clearly $\alpha_{D^{*}}=\alpha_{D}^{-1} \in \mathscr{G}(\mathscr{D})$ if $\alpha_{D} \in \mathscr{G}(\mathscr{D})$. Next, the unit element $e=\alpha_{0} \in \mathscr{G}(\mathscr{D})$. Now let $\alpha_{D}, \alpha_{E} \in \mathscr{G}(\mathscr{D})$. Then for $A \in \mathscr{F}$, 
$\left(\alpha_{D} \circ \alpha_{E}\right)[A]=(\mathbb{1}+F) A\left(\mathbb{1}+F^{*}\right)$, where $F .=D+E+D E \in \mathscr{D}$, furthermore a little algebra verifies $F+F^{*}=-F F^{*}=-F^{*} F$, i.e. $\alpha_{F} \in \mathscr{G}(\mathscr{D})$, or $\alpha_{D} \circ \alpha_{E} \in \mathscr{G}(\mathscr{D})$. Thus $\mathscr{G}(\mathscr{D})$ is a group. Let us consider the infinitesimal transformations of $\mathscr{G}(\mathscr{D})$ : choose for $\varepsilon$ small, the form:

$$
\alpha_{i \varepsilon D}[A]=(\mathbb{1}+i \varepsilon D) A\left(\mathbb{1}-i \varepsilon D^{*}\right) \cong A+i \varepsilon\left(D A-A D^{*}\right) .
$$

For $\alpha_{i \varepsilon D} \in \mathscr{G}(\mathscr{D})$, we should have $i \varepsilon D-i \varepsilon D^{*}=-\varepsilon^{2} D D^{*}$, i.e. $D \cong D^{*}$ or $D \in \mathscr{D}_{h}$. Since $\mathscr{G}(\mathscr{D})$ is a group, we can successively apply $\alpha_{i \varepsilon D}$ to get for $D \in \mathscr{D}_{h}$ :

$$
\begin{aligned}
\alpha_{D, \lambda}[A] & =\lim _{N \rightarrow \infty} \alpha_{i \lambda D / N}^{N}[A]=\lim _{N \rightarrow \infty}(\mathbb{1}+i \lambda D / N)^{N} A(\mathbb{1}-i \lambda D / N)^{N} \\
& =e^{i \lambda D} A e^{-i \lambda D} .
\end{aligned}
$$

Thus $\alpha_{D, \lambda} \in \mathscr{G}(\mathscr{D})$ or $\mathscr{G}_{u}(\mathscr{D}) \subset \mathscr{G}(\mathscr{D})$. Whilst $\mathscr{G}_{u}(\mathscr{D})$ and $\mathscr{G}(\mathscr{D})$ have the same infinitesimal generators, we cannot prove that they are equal because $\mathscr{G}_{u}(\mathscr{D})$ is arcwise connected, but $\mathscr{G}(\mathscr{D})$ is not obviously so, i.e. if we try to construct the arc $\alpha_{\lambda D}, \lambda \in[0,1]$ connecting $e$ to $\alpha_{D}$, then we find that $\lambda D+\lambda D^{*}=\lambda^{2} D D^{*}=-\lambda^{2} D^{*} D$ can only be satisfied for $\lambda=0,1$, i.e. $\alpha_{\lambda D} \notin \mathscr{G}(\mathscr{D})$ for $\lambda \in(0,1)$. This is because the set $\left\{D \in \mathscr{D} \mid D+D^{*}=-D D^{*}=-D^{*} D\right\}$ is a group under the composition law $\mathrm{D}_{1} \circ D_{2}=D_{1}+D_{2}+D_{1} D_{2}$, but is not closed under scalar multiplication.

(ii) $\mathbf{L}_{i}(\lambda) \in \mathscr{A}(\mathbf{L}) \subset \mathscr{D}$. Thus

$$
\alpha_{i, \lambda}[A]=\mathbf{U}_{i}(\lambda) A \mathbf{U}_{i}(\lambda)^{-1}=\left(\mathbb{1}+\mathbf{L}_{i}(\lambda)\right) A\left(\mathbb{1}+\mathbf{L}_{i}^{*}(\lambda)\right)=\alpha_{\mathbf{L}_{i}(\lambda)}[A],
$$

where $\alpha_{\mathbf{L}_{i}(\lambda)} \in \mathscr{G}(\mathscr{D})$ because

$$
\mathbf{L}_{i}(\lambda)+\mathbf{L}_{i}^{*}(\lambda)+\mathbf{L}_{i}(\lambda) \mathbf{L}_{i}^{*}(\lambda)=0 \forall i \in I, \lambda \in \mathbb{R} .
$$

Thus $\mathscr{G}(\chi) \subset \mathscr{G}(\mathscr{D})$. Let $\alpha_{D} \in \mathscr{G}(\mathscr{D})$. Then

$$
\alpha_{D}[A]=(\mathbb{1}+D) A\left(\mathbb{1}+D^{*}\right)=A+D A+A D^{*}+D A D^{*}=A+D_{D}(A),
$$

where the last relation defines $D_{D}(A)$. It is clear that $D_{D}: \mathcal{O} \rightarrow \mathscr{D}$ is a linear map, and that

$$
D_{D}(A B)=A D_{D}(B)+D_{D}(A) B+D_{D}(A) D_{D}(B),
$$

as can be seen by expansion. Since $D_{D}\left(A^{*}\right)=D_{D}(A)^{*}$, the conditions of Theorem 3.3 (iii) are fulfilled, and $\alpha_{D} \in \operatorname{Ker} T$, i.e. $\mathscr{G}(\mathscr{D}) \subseteq \operatorname{Ker} T$.

Lemma $\left\{x \in \mathcal{O} \mid x \mathcal{O} \subset \mathscr{K} \supset x^{*} \mathcal{O}\right\} \subset \mathscr{D}$, where $\mathscr{K}:=\cap\left\{\operatorname{Ker} \omega \mid \omega \in \mathbb{S}_{D}\right\}$.

Proof. Let $\mathscr{A}(\cdot)$ denote the $C^{*}$-algebra in $\mathcal{O}$ generated by its argument. Since $\mathcal{O}$ is a unital $C^{*}$-algebra, $\mathscr{A}(x) \subseteq \mathscr{A}(x, \mathbb{1}) \subset \mathcal{O} \forall x \in \mathcal{O}$. Suppose that for an $x \in \mathcal{O}$ we have $x \mathcal{O}$ $\subset \mathscr{K} \supset x^{*} \mathcal{O}$. Then $x \mathscr{A}(x, \mathbb{1}) \subset x \mathscr{O} \subset \mathscr{K}$, and $x^{*} \mathscr{A}(x, \mathbb{1}) \subset x^{*} \mathcal{O} \subset \mathscr{K}$, and thus $x \mathscr{A}(x, \mathbb{1})$ $+x^{*} \mathscr{A}(x, \mathbb{1})=\mathscr{A}(x) \subset \mathscr{K}$, as $\mathscr{K}$ is a linear space. But by Theorem $2.13, \mathscr{D}$ is the largest $C^{*}$-algebra in $\mathscr{K}$ in the sense that it contains all $C^{*}$-algebras contained in $\mathscr{K}$. Thus $x \in \mathscr{A}(x) \subset \mathscr{D}$.

Theorem 3.8. $\operatorname{Ker} T=\left\{\alpha_{g} \in \operatorname{Aut} \mathscr{F} \mid\left\langle\omega, \alpha_{g}[A] F\right\rangle=\langle\omega, A F\rangle \forall \omega \in \mathfrak{S}_{D} ; \forall A, F \in \mathcal{O}\right\}$.

Proof. It is clear from Theorem 3.3 (iii) that if $\alpha_{g} \in \operatorname{Ker} T$, then

$$
\left\langle\omega, \alpha_{g}[A] F\right\rangle=\langle\omega, A F\rangle \forall \omega \in \mathfrak{S}_{D} ; A, F \in \mathcal{O} .
$$


Conversely, let $\alpha_{g} \in$ Aut $\mathscr{F}$ such that

$$
\left\langle\omega, \alpha_{g}[A] F\right\rangle=\langle\omega, A F\rangle \forall \omega \in \subseteq_{D}, \forall F, A \in \mathcal{O} .
$$

Then

$$
\left(\alpha_{g}[A]-A\right) F \in \cap\left\{\operatorname{Ker} \omega \mid \omega \in \subseteq_{D}\right\}=\mathscr{K} \forall F, A \in \mathcal{O},
$$

i.e. $\left(\alpha_{g}[A]-A\right) \mathcal{O} C \mathscr{K} \forall A \in \mathcal{O}$, and since $\alpha_{g}$ is a *automorphism, and $\mathcal{O}$ is a $C^{*}$ algebra, $\left(\alpha_{g}[A]-A\right)^{*} \mathscr{O} \subset \mathscr{K} \forall A \in \mathcal{O}$. Thus by the lemma above: $\alpha_{g}[A]$ $-A \in \mathscr{D} \forall A \in \mathcal{O}$, i.e. $\alpha_{g}[A]=A+d_{g}(A)$, where $d_{g}: \mathcal{O} \rightarrow \mathscr{D}$ is an involutive, linear map satisfying

$$
d_{g}(A B)=d_{g}(A) B+A d_{g}(B)+d_{g}(A) d_{g}(B) \forall A, B \in \mathcal{O} .
$$

This property derives from the fact that $\alpha_{g}$ is a *-homomorphism. It then follows from Theorem 3.3 (iii) that $\alpha_{g} \in \operatorname{Ker} T$.

Since $\mathscr{R}$ is interpreted as the algebra of the physical observables, the implication of Theorems 3.3 and 3.8 is that all the transformations on $\mathscr{F}$ which have physical significance must be in $\mathfrak{P}$, and the gauge transformations are in $\operatorname{Ker} T$. By Theorem 3.3 (ii) we have that $T(\mathfrak{P})$ is only included in $\operatorname{Aut}(\mathscr{R})$ and is not equal to it. This is related to the problem of "lifting" an automorphism from a factor algebra to the algebra from which it is constructed, which is still an unsolved problem [25], and it is also related to the problem of extending automorphisms from a subalgebra to a larger algebra which is not always possible. Therefore, there are usually automorphisms on the physical algebra $\mathscr{R}$ which cannot be derived from automorphisms on $\mathscr{F}$, i.e. are not contained in $T(\mathfrak{P})$. We therefore do not generally expect that all physical transformations can be defined on either $\mathscr{F}$ or $\mathcal{O}$, but if they can be defined on $\mathscr{F}$, they should be in $\mathfrak{P}$.

The situation as described above gives a particular choice of structure, i.e. given a set of constraints $\left\{\mathbf{U}_{i}(\lambda)\right\}$, we can define the physical observable algebra $\mathscr{R}$, and the set of physical transformations $\mathfrak{B}$ on $\mathscr{F}$. In practice however, one is faced with a different situation; along with the set $\left\{\mathbf{U}_{i}(\lambda)\right\}$, one is also given a subset of the set of physical transformations of the system, e.g. a realization of the Lorentz group. Call this subset $\mathfrak{P}_{1}$. In this case we would first have to verify that $\mathfrak{P}_{1} \subseteq \mathfrak{P}$, before being able to claim that $\mathscr{R}$ is the physical observable algebra. When this does not hold, i.e. $\exists \alpha \in \mathfrak{P}_{1}$ such that $\alpha[\mathscr{D}] \neq \mathscr{D}$, we would have to alter the procedure above.

There are various ways in which $\alpha[\mathscr{D}]=\mathscr{D}$ can fail. First, consider $\alpha[\mathscr{D}] \nsupseteq \mathscr{D}$. This means that we would have to augment the system with "secondary quantum constraints," i.e. additional constraints generated by the application of $\mathfrak{P}_{1}$ to the original constraint set. Then one would have to impose as constraints the $\mathfrak{P}_{1}$-orbit set of the original constraints $\left\{\mathbf{U}_{i}(\lambda)\right\}$, i.e. the set: $\left\{\alpha\left[\mathbf{U}_{i}(\lambda)\right] \mid \alpha \in \mathfrak{P}_{1}, i \in I, \lambda \in \mathbb{R}\right\}$. The $T$-procedure is now applied as before, providing that $\mathbb{1} \notin \mathscr{A}\left(\mathbf{L}_{1}\right)$, where $\mathscr{A}\left(\mathbf{L}_{1}\right)$ is the $C^{*}$-algebra generated from the $\mathfrak{P}_{1}$-orbit set of $\left\{\mathbf{L}_{i}(\lambda)\right\}$. The resultant algebra $\mathscr{R}_{1}$ can now be interpreted as the physical observable algebra if we can show that $\alpha\left[\mathscr{D}_{1}\right]=\mathscr{D}_{1} \forall \alpha \in \mathfrak{P}_{1}$. This might not be generally the case, although it certainly is when $\mathfrak{P}_{1}$ is one dimensional, e.g. time translation, since then $\alpha\left[\mathscr{A}\left(\mathbf{L}_{1}\right)\right]=\mathscr{A}\left(\mathbf{L}_{1}\right)$, $\forall \alpha \in \mathfrak{P}_{1}$. When we find that $\alpha\left[\mathscr{D}_{1}\right] \neq \mathscr{D}_{1}$ for some $\alpha \in \mathfrak{P}_{1}$, we can only conclude that the constraint set $\left\{\mathbf{U}_{i}(\lambda)\right\}$ and the given set of transformations $\mathfrak{P}_{1}$ are inconsistent, and a different choice of constraints or transformations $\mathfrak{P}_{1}$ is called for. 
If $\alpha[\mathscr{D}] \subsetneq \mathscr{D}$ for some $\alpha \in \mathfrak{B}_{1}$, it means that there are too many gauge transformations, i.e. some are eliminated by physical transformations, or in other words, the set $\left\{\mathbf{U}_{i}(\lambda)\right\}$ is too large. To see this, consider the earlier observation that $\alpha[\mathscr{A}(\mathbf{L})]=\mathscr{A}(\mathbf{L}) \Rightarrow \alpha[\mathscr{D}]=\mathscr{D}$, the fact that $\mathscr{A}(\mathbf{L})$ is generated by $\left\{\mathbf{L}_{i}(\lambda)\right\}$ and that $\alpha$ is a homomorphism. Then we get that

$$
\alpha[\mathscr{D}] \subsetneq \mathscr{D} \Leftrightarrow \alpha\left[\left\{\mathbf{L}_{i}(\lambda)\right\}\right] \subsetneq\left[\left\{\mathbf{L}_{i}(\lambda)\right\}\right],
$$

i.e. the constraint set is mapped onto a smaller subset. A natural candidate for the new constraint set could be:

$$
\mathscr{L}=\bigcup_{\alpha \in \mathfrak{P}_{1}} \alpha\left[\bigcap_{\alpha \in \mathfrak{P}_{1}} \alpha\left[\left[\left\{\mathbf{L}_{i}(\lambda)\right\}\right]\right]\right],
$$

but again, this will only give a consistent result through the $T$-procedure if $\mathbb{1} \notin \mathscr{A}(\mathscr{L})$ and $\alpha\left[\mathscr{D}_{\mathscr{L}}\right]=\mathscr{D}_{\mathscr{L}} \forall \alpha \in \mathfrak{P}_{1}$ and the notation is obvious.

In the case when $\alpha[\mathscr{D}]-\mathscr{D} \neq \emptyset \neq \mathscr{D}-\alpha[\mathscr{D}]$ for some $\alpha \in \mathfrak{P}_{1}$, it seems we will have to choose a new constraint set to make the system consistent, and it seems easiest to choose

$$
\mathscr{L} .=\bigcup_{\alpha \in \mathfrak{P}_{1}} \alpha\left[\bigcap_{\alpha \in \mathfrak{P}_{1}} \alpha[\mathscr{D}]\right] .
$$

As before, this is a matter of choice, and the consistency conditions given above will have to hold.

\section{Algebraic Conditions}

In Assumption 2.2 we also assumed the existence of algebraic conditions $\left\{\mathbf{V}_{i}(\lambda)\right\}$. Define $\mathbf{N}_{i}(\lambda)=\mathbf{V}_{i}(\lambda)-\mathbb{1}$, and let $\mathscr{A}(\mathbf{N})$ be the $C^{*}$-algebra generated in $\mathscr{F}$ by the set $\left\{\mathbf{N}_{i}(\lambda)\right\}$. In heuristic theories, algebraic conditions are often imposed in the form $\xi_{r}=0$. In this framework it does not make sense to put directly $N=0 \forall N \in \mathscr{A}(\mathbf{N})$, because these elements in the $C^{*}$-algebra $\mathscr{F}$ are not zero. We would have to impose some construction wherein $\mathscr{A}(\mathbf{N})=0$ holds. In some theories (e.g. if the constraints are a pair of conjugate variables $(p, q))$, we have the structure that $\mathscr{F}=\mathscr{A}(\mathbf{N}) \otimes \mathscr{R}$, where $\mathscr{R}$ is a $C^{*}$-algebra. In this case topological arguments, aside, it is very simple to omit $\mathscr{A}(\mathbf{N})$ from the framework by selecting $\mathscr{R}$ as the physical observable algebra. In more difficult situations where we do not have this direct product structure, but where $\mathscr{F}$ is a von Neumann algebra, we can make use of the spectral projections of the constraints. Let $P_{N}$ be the domain projection of $N \in \mathscr{A}(\mathbf{N})$, then we select the set of observables by $\mathcal{O} .=\left\{A \in \mathscr{F} \mid A P_{N}=0=P_{N} A \forall N \in \mathscr{A}(\mathbf{N})\right\}$. This definition makes sense since $\mathscr{F}$ is a von Neumann algebra and hence $P_{N} \in \mathscr{F}$. Notice that $\mathcal{O}$ is empty whenever $\mathbb{1} \in \mathscr{A}(\mathbf{N})$, so that if $\mathscr{A}(\mathbf{N})$ derives from a set of second class Dirac constraints, this is very likely.

In the case when $\mathscr{F}$ is a general $C^{*}$-algebra, we cannot use the method above because we cannot be sure that $P_{N} \in \mathscr{F} \forall N \in \mathscr{A}(\mathbf{N})$. A natural generalization of the condition above would be $A \in \mathcal{O}$ if $A N=0=N A \forall N \in \mathscr{A}(\mathbf{N})$, but this seems unduly strict because then $\mathcal{O}$ will be a very small subalgebra of $\mathscr{A}(\mathbf{N})^{\prime}$, which is the traditional observables. To generalize the condition, observe that all we need to require is that $\mathscr{A}(\mathbf{N})$ acts as a zero element on $\mathcal{O}$, i.e. $A N \in \mathscr{A}(\mathbf{N})$, 
$N A \in \mathscr{A}(\mathbf{N}) \forall N \in \mathscr{A}(\mathbf{N}), A \in \mathcal{O}$. From this it is clear that $\mathcal{O}$ is the multiplier algebra of $\mathscr{A}(\mathbf{N})$, i.e. $\mathcal{O}_{a}=M(\mathscr{A}(\mathbf{N}))$ [cf. (2.9)]. Then $\mathscr{A}(\mathbf{N})$ will be a proper ideal of $\mathcal{O}_{a}$ iff $\mathbb{1} \notin \mathscr{A}(\mathbf{N})$, and this will be the nontriviality condition for the system, again casting doubt on deriving $\mathscr{A}(\mathbf{N})$ from second class Dirac constraints. Then we can define the algebra of physical observables as $\mathscr{R}_{a}=\mathcal{O}_{a} / \mathscr{A}(\mathbf{N})$.

The algebraic construction above seems simple and natural until one considers the states on $\mathscr{R}_{a}$. By Dixmier 2.11.6 [18], there is a canonical bijection between the states on $\mathscr{R}_{a}$ and the states on $\mathcal{O}_{a}=M(\mathscr{A}(\mathbf{N}))$, such that $\mathscr{A}(\mathbf{N}) \subset \operatorname{Ker} \omega$. This means that through the construction above, we are imposing essentially the same type of requirement as the state conditions, and so the same theory as in Sect. 2 would apply. In other words, to put $\mathscr{A}(\mathbf{N})$ equal to zero is the same as putting [ $\mathscr{F} \mathscr{A}(\mathbf{N})]$ $\cap[\mathscr{A}(\mathbf{N}) \mathscr{F}]=\mathscr{D}_{a}$ equal to zero. So for the total system, the $\left\{\mathbf{L}_{i}(\lambda)\right\}$ and $\left\{\mathbf{N}_{i}(\lambda)\right\}$ are to be treated on the same footing. Work is in progress to prove that $\mathscr{R}_{a}$ is isomorphic to $\mathscr{R}=M\left(\mathscr{D}_{a}\right) / \mathscr{D}_{a}$. If $\mathscr{A}$ is the $C^{*}$-algebra generated by $\mathscr{A}(\mathbf{L})$ and $\mathscr{A}(\mathbf{N})$, the nontriviality condition would then be $\mathbb{1} \notin \mathscr{A}$.

If one chose to quantise Dirac's second class constraints as algebraic conditions, it seems unlikely that $\mathbb{1} \notin \mathscr{A}$, for the following reasons. The second class constraints are phase space functionals whose mutual Poisson brackets do not lie in the linear space generated by the full set of constraints. So if one quantises in such a way that the second class constraints are nonzero elements of the field algebra, it is unlikely that their mutual commutators will always lie in the algebra generated by the first class constraints. In fact, if one of the commutators gives rise to an invertible element, then the generated algebra will be unital. A simple example of this is to choose a pair of canonical vairables $(q, p)$ as second class constraints. Then $\mathbb{1} \in \mathscr{A}(q, p)$. It is not difficult to construct examples of degenerate Lagrangians with constraints such that $\mathbb{1} \in \mathscr{A}$. Thus if we wish to apply the construction above, the best one can do is to follow the spirit of Dirac's suggestion by quantizing only the reduced phase space functionals, possibly in natural coordinates, in which case the second-class constraints do not enter the quantum theory at all. We are considering alternative algebraic constructions for eliminating constraint algebras $\mathscr{A}(\mathbf{N})$ such that $\mathbb{1} \in \mathscr{A}(\mathbf{N})$, canonically from the theory, and may pursue this further in future publications.

\section{An Example: Electromagnetism}

The classical form of electromagnetism under consideration is given by $S=-\frac{1}{4} \int F_{\mu \nu} F^{\mu \nu} d^{4} x$, where $F_{\mu v}(x)=A_{v, \mu}(x)-A_{\mu, v}(x)$, which results in the EulerLagrange equation $F_{\mu \nu}{ }^{, v}(x)=0$. The momenta conjugate to the vector potentials are $B_{\mu}(x)=F_{\mu 0}(x)$, and thus the equal-time Poisson bracket relations hold:

$$
\begin{aligned}
& \left\{A_{\mu}(x), A_{v}\left(x^{\prime}\right)\right\}_{x_{0}=x_{0}^{\prime}}=\left\{B_{\mu}(x), B_{v}\left(x^{\prime}\right)\right\}_{x_{0}=x_{0}^{\prime}}=0 \\
& \left\{A_{\mu}(x), B_{v}\left(x^{\prime}\right)\right\}_{x_{0}=x_{0}^{\prime}}=g_{\mu \nu} \delta^{3}\left(\mathbf{x}-\mathbf{x}^{\prime}\right) .
\end{aligned}
$$

It is easy to see that the Lagrangian is degenerate, and it gives rise to the two constraints $B_{0}(x) \approx 0$ and $B_{r}{ }^{r}(x) \approx 0$, and these are first-class.

In quantizing this system, we use some methods of [16], and the general framework of [22]. First we need to smear the $A_{\mu}(x), B_{\mu}(x)$ with suitable test 
functions. From the CCR we see that we need to retain the sharp time aspect, and thus we smear according to

$$
A_{x_{0}}(F) .=\int d^{3} x A_{\mu}\left(x_{0}, \mathbf{x}\right) f^{\mu}(\mathbf{x}), \quad B_{x_{0}}(F)=\int d^{3} x B_{\mu}\left(x_{0}, \mathbf{x}\right) f^{\mu}(\mathbf{x}),
$$

where $F(\mathbf{x})=\left(f^{0}(\mathbf{x}), f^{1}(\mathbf{x}), f^{2}(\mathbf{x}), f^{3}(\mathbf{x})\right)$ is a 4-component vector where each component belongs to the space of Schwarz functions on $\mathbb{R}^{3}, \mathscr{S}\left(\mathbb{R}^{3}\right)$, i.e. they are real valued, infinitely differentiable and of fast decrease. In other words, $F \in \sum_{i=0}^{3} \oplus \mathscr{S}^{(i)}\left(\mathbb{R}^{3}\right)$. Then the CCR are:

and

$$
\left[A_{x_{0}}(F), A_{x_{0}}(G)\right]=0=\left[B_{x_{0}}(F), B_{x_{0}}(G)\right],
$$

$$
\left[A_{x_{0}}(F), B_{x_{0}}(G)\right]=i \int d^{3} x f^{\mu}(\mathbf{x}) g_{\mu}(\mathbf{x})=. i(F, G) .
$$

To form the $C^{*}$-algebra of the CCR, (cf. $\left.[14,22]\right)$, we need a pair $(\mathscr{Q}, B)$, where $\mathscr{Q}$ is a locally convex topological vector space, and $B(\cdot, \cdot)$ is a nondegenerate antisymmetric continuous bilinear form on $\mathscr{Q}$. Now for $\mathscr{Q}$, we choose the complexification of the test function space, i.e.

$$
\mathscr{Q}=\sum_{i=0}^{3} \oplus \mathscr{S}^{(i)}\left(\mathbb{R}^{3}\right)+i \sum_{i=0}^{3} \oplus \mathscr{S}^{(i)}\left(\mathbb{R}^{3}\right),
$$

which will be a locally convex vector space with the topology induced by the usual norm:

$$
\left\|F_{1}+i F_{2}\right\|=\left(\sum_{\mu=0}^{3}\left\|f_{1}^{\mu}\right\|^{2}+\sum_{\mu=0}^{3}\left\|f_{2}^{\mu}\right\|^{2}\right)^{1 / 2},
$$

where $\left\|f^{\mu}\right\|$ is the supremum norm, and $F=F_{1}+i F_{2} \in \mathscr{Q}$. For the symplectic form $B(\cdot, \cdot)$ we follow the literature (i.e. [23]) and choose

$$
B(F, G)=B\left(F_{1}+i F_{2}, G_{1}+i G_{2}\right)=\left(F_{1}, G_{2}\right)-\left(F_{2}, G_{1}\right) .
$$

This form is bilinear, continuous and antisymmetric. That it is also nondegenerate is easily verifiable.

Definition 5.1. Let $\Delta(2)$ be the normed *-algebra such that:

(i) The elements of $\Delta(\mathscr{Q})$ are complex valued functions on $\mathscr{2}$ with support consisting of a finite subset of 2 .

(ii) Let $\Delta(\mathscr{Q})$ have the obvious linear structure, and the multiplication law:

$$
\left(f_{1} f_{2}\right)(z)=\sum_{z_{1} \in \mathscr{2}} f_{1}\left(z_{1}\right) f_{2}\left(z-z_{1}\right) \exp \left[-i B\left(z_{1}, z\right) / 2\right]
$$

The involution is defined by $f^{*}(z)=\overline{f(-z)}$.

(iii) Define a norm in $\Delta(\mathscr{Q})$ by $\|f\|_{1}=\sum_{z \in \mathscr{Q}}|f(z)|$.

The completion in this norm of $\Delta(\mathscr{Q})$ is denoted $\Delta_{1}(\mathscr{Q})$. The set of functions $\delta_{z}$, such that $\delta_{z}\left(z^{\prime}\right)=1$ if $z=z^{\prime}$ and zero otherwise, forms a linear basis for $\Delta(\mathscr{Q})$. Let $P$ denote the set of all nondegenerate representations $\pi$ of $\Delta_{1}(\mathscr{Q})$ by bounded Hilbert space operators, such that $\pi\left(\delta_{\lambda z}\right)$ is weakly continuous in $\lambda \in \mathbb{R} \forall z \in \mathscr{Q}$.

Definition 5.2. The $C^{*}$-algebra of the CCR over $(\mathscr{Q}, B)$, is the completion of $\Delta_{1}(\mathscr{Q})$ in the norm $\|f\|=\sup _{\pi \in P}\|\pi(f)\|$, and is denoted by $\overline{\Delta(\mathscr{Q})}$. 
We shall choose this as our field algebra, i.e. $\mathscr{F}=\overline{\Delta(\mathscr{Q})}$. In order to apply the constraint formalism of Sect. 2, we need to define the set $\left\{\mathbf{U}_{i}(\lambda)\right\}$. Given a pair $(\mathscr{Q}, B)$ as specified above, a Weyl system over $(\mathscr{Q}, B)$ is defined as a map $W$ from $\mathscr{Q}$ into the set of unitary operators on a Hilbert space, such that $W(\lambda z)$ is strongly continuous in $\lambda \in \mathbb{R}$ for fixed $z \in \mathscr{Q}$, and the multiplication law

$$
W(z) W\left(z^{\prime}\right)=W\left(z+z^{\prime}\right) \exp [-i B(z, z) / 2]
$$

is satisfied. Now a Weyl system is postulated to arise via

where formally

$$
W(F)=U\left(F_{1}\right) V\left(F_{2}\right) \exp \left[-i\left(F_{1}, F_{2}\right) / 2\right],
$$

$$
U(F)=\exp \left(i B_{x_{0}}(F)\right) ; \quad V(G)=\exp \left(i A_{x_{0}}(G)\right) .
$$

We would like to pursue this formal aspect further in order to define the quantum constraints, where the link with $\mathscr{F}$ is furnished by the result [14], that there is a bijection between the set of all representations $\pi \in P$, and the Weyl systems on $(\mathscr{Q}, B)$, and this is realized by the relation: $W_{\pi}(F)=\pi\left(\delta_{F}\right), F \in \mathscr{Q}, \pi \in P$.

Let $F=(f(\mathbf{x}), 0,0,0), f(\mathbf{x}) \in \mathscr{S}\left(\mathbb{R}^{3}\right)$. Then $B_{x_{0}}(F)=\int d^{3} x B_{0}\left(x_{0}, \mathbf{x}\right) f(\mathbf{x})$, and this is a smeared first constraint $B_{0}$. For the second constraint $B_{r}{ }^{r}$, consider

$$
F=\left(0,-f(\mathbf{x})_{, 1},-f(\mathbf{x})_{, 2},-f(\mathbf{x})_{, 3}\right) \in \sum_{i=0}^{3} \oplus \mathscr{S}^{(i)}\left(\mathbb{R}^{3}\right) .
$$

Then $B_{x_{0}}(F)=\int d^{3} x B_{r},{ }^{r}\left(x_{0}, \mathbf{x}\right) f(\mathbf{x})$. Therefore form the two classes of test functions:

$$
\begin{gathered}
\mathscr{C}_{1}=\left\{F \in \sum_{i=0}^{3} \oplus \mathscr{S}^{(i)}\left(\mathbb{R}^{3}\right) \mid F=(f(\mathbf{x}), 0,0,0)\right\}+i(0,0,0,0) \subset \mathscr{Q} \\
\mathscr{C}_{2}=\left\{F \in \sum_{i=0}^{3} \oplus \mathscr{S}^{(i)}\left(\mathbb{R}^{3}\right) \mid F=\left(0,-f(\mathbf{x})_{, 1},-f(\mathbf{x})_{, 2},-f(\mathbf{x})_{, 3}\right)\right\}+i(0,0,0,0) \subset \mathscr{Q} .
\end{gathered}
$$

Thus in consideration of the results mentioned beforehand, formally $\left\{W(F) \mid F \in \mathscr{C}_{1}\right\}$ corresponds to

$$
\left\{\exp \left[i \int B_{0}\left(x_{0}, \mathbf{x}\right) f(\mathbf{x}) d^{3} x\right]\right\},
$$

and $\left\{W(F) \mid F \in \mathscr{C}_{2}\right\}$ corresponds to

$$
\left\{\exp \left[i \int B_{r}{ }^{r}\left(x_{0}, \mathbf{x}\right) f(\mathbf{x}) d^{3} x\right] \mid f(\mathbf{x}) \in \mathscr{S}\left(\mathbb{R}^{3}\right)\right\} .
$$

Then using the relation $W_{\pi}(F)=\pi\left(\delta_{F}\right)$, it is reasonable to define $\mathbf{U}_{i}=\left\{\delta_{F} \mid F \in \mathscr{C}_{i}\right.$; $i=1,2\}$ as the $\mathbf{U}_{i}$ of the state conditions. Note that $\mathscr{C}_{1} \cap \mathscr{C}_{2}=\{0\}$, and thus $\mathbf{U}_{1} \cap \mathbf{U}_{2}$ $=\delta_{0}=\mathbb{1}$.

This method of defining constraints by selecting sets of test functions will apply to any problem of bosons with linear constraints. However, if the constraints are nonlinear, one might have to use a more elaborate method, like defining the $\mathbf{U}_{i}(\lambda)$ in terms of series or integrals of the $\delta_{F}$.

Now that we have defined $\mathscr{F}$ and $\mathbf{U}_{i}$, the procedure of Sect. 2 can be applied. Define $\mathbf{L}_{i}(\lambda F)=\delta_{\lambda F}-\mathbb{1}, \lambda \in \mathbb{R}, F \in \mathscr{C}_{i} ; i=1,2$. Then $\mathscr{A}(\mathbf{L})$ is the $C^{*}$-algebra generated by all the $\mathbf{L}_{i}(\lambda F)$. The Dirac states are defined by

$$
\omega \in \mathfrak{S}_{D} \quad \text { iff }\left\langle\omega, \delta_{\lambda F} A\right\rangle=\langle\omega, A\rangle=\left\langle\omega, A \delta_{\lambda F}\right\rangle \forall A \in \mathscr{F}, \lambda \in \mathbb{R}, F \in \mathscr{C}_{i}
$$


We have used here the equivalent condition mentioned in Sect. 2. Then choose $A=\delta_{f}, f \in \mathscr{Q}$, to get:

$$
\begin{aligned}
\omega \in \mathfrak{S}_{D} \text { iff }\left\langle\omega, \delta_{f}\right\rangle & =\left\langle\omega, \delta_{\lambda F+f}\right\rangle \exp [-i B(\lambda F, f) / 2] \\
& =\left\langle\omega, \delta_{\lambda F+f}\right\rangle \exp [i B(\lambda F, f) / 2],
\end{aligned}
$$

where use was made of the multiplication rules. For a fixed $f \in \mathscr{Q}$, this equality can only hold if either

$$
\left\langle\omega, \delta_{\lambda F+f}\right\rangle=0 \forall \lambda \in \mathbb{R}, F \in \mathscr{C}_{i}, \omega \in \mathfrak{S}_{D},
$$

or/and if $B(\lambda F, f)=2 n \pi \forall \lambda \in \mathbb{R}, F \in \mathscr{C}_{i}$ and some $n \in \mathbb{Z}$. In the first case we find $\left\langle\omega, \delta_{f}\right\rangle=0 \forall \omega \in \mathfrak{S}_{D}$, i.e. $\delta_{f} \in \mathscr{K}=\cap\left\{\operatorname{Ker} \omega \mid \omega \in \mathfrak{S}_{D}\right\}$. In the second case we find $B(F, f)=0 \forall F \in \mathscr{C}_{i}$, i.e. via multiplication rule, $\delta_{f} \in \mathscr{A}(\mathbf{L})^{\prime}$. So for any $f \in \mathscr{Q}$, we have either $\delta_{f} \in \mathscr{K}$, or/and $\delta_{f} \in \mathscr{A}(\mathbf{L})^{\prime}$, i.e. the Dirac states will vanish on all those $\delta_{f}$ which are not in the traditional observables $\mathscr{A}(\mathbf{L})^{\prime}$. Since $\left\{\delta_{f} \mid f \in \mathscr{Q}\right\}$ forms a linear basis for $\Delta(\mathscr{Q})$, this implies a very simple structure for $\Delta(\mathscr{Q})$, i.e. every $A \in \Delta(\mathscr{Q})$ can be written as $A=k+p$, where

and

$$
k=\sum_{i=1}^{n} \lambda_{i} \delta_{f_{i}}, n<\infty, \lambda_{i} \in \mathbb{C}, \delta_{f_{i}} \in \mathscr{K},
$$

$$
p=\sum_{i=1}^{m} \gamma_{i} \delta_{g_{i}}, m<\infty, \gamma_{i} \in \mathbb{C}, \delta_{g_{i}} \in \mathscr{A}(\mathbf{L})^{\prime}
$$

This means that $\mathscr{Q}=k \cup \not p$, where $k=\left\{f \in \mathscr{Q} \mid \delta_{f} \in \mathscr{K}\right\}$ and $\not==\left\{f \in \mathscr{Q} \mid \delta_{f} \in \mathscr{A}(\mathbf{L})^{\prime}\right\}$, and $\Delta(\mathscr{Q})=K+P$, where $K$ (respectively $P$ ) is the linear space generated by $\left\{\delta_{f} \mid f \in k\right\}$ (respectively $\left\{\delta_{f} \mid f \in \not h\right\}$ ). $P$ is a $*$-algebra. Furthermore, $\left\{\delta_{F} \mid F \in \mathscr{C}_{i}\right\} \subset P$, and since $\mathbb{1} \in P$, we find the ${ }^{*}$-algebra generated by $\mathbf{L}_{i}(\lambda F)$ in $\Delta(\mathscr{Q})$, denoted by $\mathscr{A}_{0}(\mathbf{L})$, to satisfy $\mathscr{A}_{0}(\mathbf{L}) \subset K \cap P$. Since $\mathbf{L}_{i}(\lambda F) \in P \subset \mathscr{A}(\mathbf{L})^{\prime}, \mathscr{A}(\mathbf{L}) \subset \mathscr{A}(\mathbf{L})^{\prime}$, it follows that $\mathscr{A}(\mathbf{L})$ is abelian. Whilst this is satisfied for electromagnetism, it is a very strong restriction in general, since it would also apply to all boson theories with linear constraints, as would all the preceding structure as well as the theory to follow. Since $\mathscr{A}(\mathbf{L})$ and thus $\mathscr{A}_{0}(\mathbf{L})$ is abelian, it follows from the multiplication rule that $\mathscr{A}_{0}(\mathbf{L})$ consists of specific linear combinations of $\left\{\delta_{F} \mid F \in\left[\mathscr{C}_{1} \cup \mathscr{C}_{2}\right]\right\}$.

In passing from $\Delta(\mathscr{Q})$ to $\mathscr{F}$, we first completed $\Delta(\mathscr{Q})$ in the $\|\cdot\|_{1}$-norm, and then completed it in the $C^{*}$-norm. Now

$$
\Delta_{1}(\mathscr{Q})=\left\{\sum_{i=0}^{\infty} \lambda_{i} \delta_{f_{i}}\left|f_{i} \in \mathscr{Q}, \lambda_{i} \in \mathbb{C}, \sum_{i=0}^{\infty}\right| \lambda_{i} \mid<\infty\right\},
$$

and we still have $\Delta_{1}(\mathscr{Q})=K_{1}+P_{1}$, where in this context we redefine

$$
\begin{aligned}
K_{1} & \left(\text { respectively } P_{1}\right) \\
& =\left\{\sum_{i=0}^{\infty} \lambda_{i} \delta_{f_{i}} \mid f_{i} \in k(\text { respectively } \not p), \lambda_{i} \in \mathbb{C}, \sum_{i=0}^{\infty}\left|\lambda_{i}\right|<\infty\right\} .
\end{aligned}
$$

Denote the closure of $K_{1}$ (respectively $\left.P_{1}\right)$ in the $C^{*}$-norm by $\Sigma$ (respectively $\Lambda$ ). Since $K_{1} \subset \mathscr{K}$ and $P_{1} \subset \mathscr{A}(\mathbf{L})^{\prime}$, and both $\mathscr{K}$ and $\mathscr{A}(\mathbf{L})^{\prime}$ are closed in the $C^{*}$-norm, it 
follows that $\Sigma \subseteq \mathscr{K}$ and $\Lambda \cong \mathscr{A}(\mathbf{L})^{\prime}$. Furthermore, since addition is continuous in the $C^{*}$-norm,

$$
\mathscr{F}=\overline{\Delta_{1}(\mathscr{Q})}=\overline{K_{1}+P_{1}}=\bar{K}_{1}+\bar{P}_{1}=\Sigma+\Lambda .
$$

$\Lambda$ is a $C^{*}$-algebra.

In order to apply the general theory we wish to construct $\mathscr{S}$. Since $\mathscr{S} C \mathscr{F}$, we have for an $s \in \mathscr{S}$ that $s=k+p$, where $k \in \Sigma, p \in \Lambda$. We need to verify $\mathscr{A}(\mathbf{L}) \mathscr{S}$ $C[\mathscr{F} \mathscr{A}(\mathbf{L})]$. Then:

$$
\left(\delta_{F}-\mathbb{1}\right) s \in[\mathscr{F} \mathscr{A}(\mathbf{L})] \forall F \in\left[\mathscr{C}_{1} \cup \mathscr{C}_{2}\right] .
$$

Since $p \in \mathscr{A}(\mathbf{L})^{\prime}$, we only need to consider $k$, and since multiplication is continuous in the $C^{*}$-norm, it suffices to consider $k \in K_{1}$, and from the form of these elements, it suffices to let $k=\delta_{f}, f \in k$. Thus we have to show $\left(\delta_{F}-\mathbb{1}\right) \delta_{f} \in[\mathscr{F} \mathscr{A}(\mathbf{L})]$. But

$$
\left(\delta_{F}-\mathbb{1}\right) \delta_{f}=\delta_{f}\left(\delta_{F} \exp [i B(f, F)]-\mathbb{1}\right),
$$

and since $\delta_{f} \in \mathscr{F}$, this will only be in $[\mathscr{F} \mathscr{A}(\mathbf{L})]$ if

$$
\left(\delta_{F} \exp [i B(f, F)]-\mathbb{1}\right) \in[\mathscr{F} \mathscr{A}(\mathbf{L})] \forall F \in\left[\mathscr{C}_{i}\right] .
$$

In this case, by Theorem 2.6 (ii) we should have $\delta_{F} \exp [i B(f, F)]-\mathbb{1} \in \mathscr{K}$, i.e.

$$
\left\langle\omega, \delta_{F} \exp [i B(f, F)]-\mathbb{1}\right\rangle=\exp [i B(f, F)]-1=0 \forall \omega \in \subseteq_{D}, F \in\left[\mathscr{C}_{i}\right] .
$$

Then $B(f, F)=0$, i.e. $\delta_{f} \in \mathscr{A}(\mathbf{L})^{\prime}$. This means that $\mathscr{S} C \mathscr{A}(\mathbf{L})^{\prime}$. Since $\mathscr{A}(\mathbf{L})^{\prime} \subset \mathscr{S} \cap \mathscr{S}^{*}$, we have $\mathscr{S}=\mathscr{S}^{*}=\mathscr{A}(\mathbf{L})^{\prime}$, and $\mathcal{O}=\mathscr{A}(\mathbf{L})^{\prime}$. Furthermore, $\mathscr{D}=\mathscr{S}^{*} \mathscr{A}(\mathbf{L}) \mathscr{\mathscr { S }}$ $=\overline{\mathscr{A}(\mathbf{L})^{\prime} \mathscr{A}(\mathbf{L})}$. So in the nontrivial case when $\mathbb{1} \notin \mathscr{A}(\mathbf{L})$, the physical observable algebra is $\mathscr{R}=\mathscr{O} / \mathscr{D}=\mathscr{A}(\mathbf{L})^{\prime} / \overline{\mathscr{A}(\mathbf{L})^{\prime} \mathscr{A}(\mathbf{L})}$.

\section{Conclusion}

This approach gives a rigorous picture for degenerate systems, but there are still some questions which need to be discussed. Firstly, the result of Gotay, Nester and Hinds [8], that the secondary first class constraints do not necessarily generate gauge transformations is not yet understood in this formalism. If Gotay [24] is correct, it seems that in the standard interpretation all first class constraints generate gauge transformations, which is what the present theory says. Secondly the explicit treatment of theories with non-linear constraints is likely to be complicated. Thirdly the verification that $\mathbb{1} \notin \mathscr{A}(\mathbf{L})$, for a given set of constraints will be difficult. The treatment here of the transformations on the algebras is incomplete, and we hope in future publications to develop this aspect further, especially in relation to the realizations of the Lorentz group, and unitary implementability.

Another aspect which needs further attention, is the presence of an indefinite inner product, especially since many degenerate systems are represented on an indefinite inner product space. There are two structural questions here. Firstly, given a field algebra that can be embedded in a $C^{*}$-algebra, and is represented on an indefinite inner product space, what can one say about these representations? This question has been addressed in [26] for a theory of bosons, where it was shown that such representations on indefinite inner product spaces are associated 
to certain nonpositive functionals on the algebra. Therefore the usual assumption that all physical information is contained in the field algebra and its set of states has to be broadened to include these nonpositive functionals, and these should also be considered in the treatment above of degenerate systems. The second structural question is, given a *-algebra of observables on an indefinite inner product space, can all the physical relevant objects in this algebra be embedded in a $C^{*}$-algebra? This question has not been addressed to the best of our knowledge, although [16] is an example where a $C^{*}$-algebra was constructed for the field algebra for a theory that is usually represented on an indefinite inner product space, electromagnetism.

\section{References}

1. Sagan, H.: Introduction to the calculus of variations. New York: McGraw-Hill 1969

Ewing, G.M.: Calculus of variations with applications. New York: Norton 1969

Choquet-Bruhat, Y., De Witt-Morette, C., Dillard-Bleick, M.: Analysis, manifolds and physics. Amsterdam: North-Holland 1982

2. Dirac, P.A.M.: Lectures on quantum mechanics. New York: Belfer Graduate School of Science, Yeshiva University 1964

Dirac, P.A.M.: Generalized Hamiltonian dynamics. Can. J. Math. 2, 129-148 (1950)

Dirac, P.A.M.: The Hamiltonian form of field dynamics. Can. J. Math. 3, 1-23 (1951)

3. Kundt, W.: Canonical quantization of gauge invariant field theories. Springer Tracts in Modern Physics, Vol. 40, pp. 107-168. Berlin, Heidelberg, New York: Springer 1966

4. Sundermeyer, K.: Constrained dynamics. In: Lecture Notes in Physics, Vol. 169. Berlin, Heidelberg, New York, Tokyo: Springer 1982

5. Hanson, A.J., Regge, T., Teitelboim, C.: Constrained Hamiltonian systems. Rome: Accademia Nazionale dei Lincei 1976

6. Sudarshan, E.C.G., Mukunda, N.: Classical dynamics: a modern perspective. New York: Wiley 1974

7. Shanmugadhasan, S.: Canonical formalism for degenerate Lagrangians. J. Math. Phys. 14, 677-687 (1975)

8. Gotay, M.J., Nester, J.M., Hinds, G.: Presymplectic manifolds and the Dirac-Bergman theory of constraints. J. Math. Phys. 19, 2388-2399 (1978)

9. Fadeev, L.D.: The Feynman integral for singular Lagrangians. Theor. Math. Phys. 1, 1-12 (1970)

Kerler, W.: Quantum treatment of constrained systems and implications for path integrals. Phys. Lett. 76 B, 423-427 (1978)

10. Bergmann, P.G., Goldberg, I.: Dirac bracket transformations in phase space. Phys. Rev. 98, 531-538 (1955)

11. Hermann, R.: Lie algebras and quantum mechanics. New York: Benjamin 1970

Boisseau, B., Barrabas, C.: Quantization of the Liouville mechanics for systems with singular Lagrangians. J. Math. Phys. 19, 1032-1036 (1978)

Castellani, L., Dominici, D., Longhi, G.: Canonical transformations and quantization of singular Lagrangian systems. Nuovo Cimento 48 A, 91-99 (1978)

12. Källen, G.: Quantum electrodynamics. London: Allan and Unwin 1972

13. Chernoff, P.R.: Mathematical obstructions to quantization. Had. J. 4, 879-898 (1981)

14. Emch, G.G.: Algebraic methods in statistical mechanics and quantum field theory. New York: Wiley 1972

15. Bratteli, O., Robinson, D.W.: Operator algebras and quantum statistical mechanics. Berlin, Heidelberg, New York: Springer 1979

16. Carey, A.L., Gaffney, J.M., Hurst, C.A.: A $C^{*}$-algebra formulation of the quantization of the electromagnetic field. J. Math. Phys. 18, 629-640 (1977) 
17. Pedersen, G.K.: $C^{*}$-algebras and their automorphism groups. London: Academic Press 1979

18. Dixmier, J.: $C^{*}$-algebras. Amsterdam: North-Holland 1977

19. Naimark, M.A.: Normed algebras. Groningen: Wolters Noordhoff 1972

20. Kapuscik, E., Uzes, C.A.: Dirac bracket quantization and central force systems. Am. J. Phys. 50, 1094-1097 (1982)

21. Kálnay, A.J.: On certain intriguing physical, mathematical and logical aspects concerning quantization. Had. J. 4, 1127-1165 (1981)

22. Manuceau, J.: $C^{*}$-algebre de relations de commutation. Ann. Inst. Henri Poincaré 8, 139-161 (1968)

23. Segal, I.E.: Mathematical problems of relativistic physics. Providence, R.I.: American Mathematical Society 1963

24. Gotay, M.J.: On the validity of Dirac's conjecture regarding first class secondary constraints. J. Phys. A 16, L 141-L 145 (1983)

25. Sullivan, D., Maitland Wright, J.D.: On lifting automorphisms. Proc. Symp. Pure Math. Am. Math. Soc., Vol. 38. Part 2, pp. 289-290 (1982)

Sullivan, D., Maitland Wright, J.D.: On lifting automorphisms of monotone $\sigma$-complete $C^{*}$ algebras. Q. J. Math. Oxford (2), 32, 371-381 (1981)

26. Jakobczyk, L.: Canonical quantization with indefinite inner product. Preprint No. 592, Institute of Theoretical Physics, University of Wroclaw, Wroclaw, Poland (1983)

Communicated by H. Araki

Received May 21, 1984; in revised form October 15, 1984 